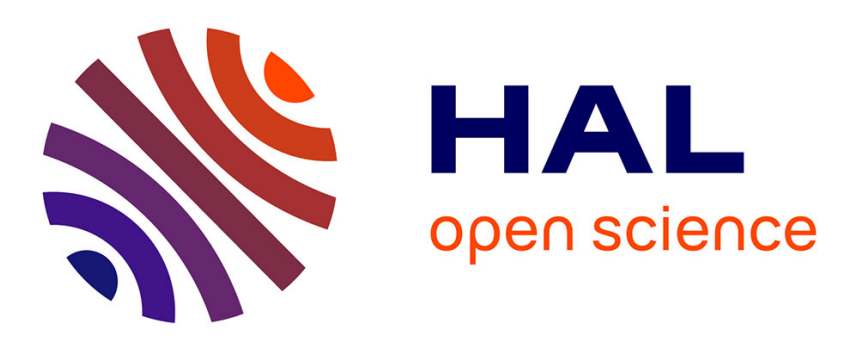

\title{
Nonsmooth Lyapunov pairs for infinite-dimensional first-order differential inclusions
}

\author{
Samir Adly, Abdelrrahim Hantoute, Michel Théra
}

\section{To cite this version:}

Samir Adly, Abdelrrahim Hantoute, Michel Théra. Nonsmooth Lyapunov pairs for infinite-dimensional first-order differential inclusions. Nonlinear Analysis: Theory, Methods and Applications, 2012, 75 (3), pp.985-1008. 10.1016/j.na.2010.11.009 . hal-00586942

\section{HAL Id: hal-00586942 https://hal.science/hal-00586942}

Submitted on 3 Apr 2019

HAL is a multi-disciplinary open access archive for the deposit and dissemination of scientific research documents, whether they are published or not. The documents may come from teaching and research institutions in France or abroad, or from public or private research centers.
L'archive ouverte pluridisciplinaire HAL, est destinée au dépôt et à la diffusion de documents scientifiques de niveau recherche, publiés ou non, émanant des établissements d'enseignement et de recherche français ou étrangers, des laboratoires publics ou privés. 


\title{
NONSMOOTH LYAPUNOV PAIRS FOR INFINITE-DIMENSIONAL FIRST-ORDER DIFFERENTIAL INCLUSIONS*
}

\author{
SAMIR ADLY ${ }^{\dagger}$, ABDERRAHIM HANTOUTE $^{\ddagger}$, AND MICHEL THERA ${ }^{\S}$
}

\begin{abstract}
The main objective of this paper is to provide new explicit criteria to characterize weak lower semicontinuous Lyapunov pairs or functions associated to first-order differential inclusions in Hilbert spaces. These inclusions are governed by a Lipschitzian perturbation of a maximally monotone operator. The dual criteria we give are expressed by the means of the proximal subdifferential of the nominal functions while primal conditions are described in terms of the Dini directional derivative. We also propose a unifying review of many other criteria given in the literature. Our approach is based on advanced tools of variational analysis and generalized differentiation.
\end{abstract}

Key words. Differential inclusions, maximal monotone operators, Lipschitz perturbations, lower semicontinuous Lyapunov pairs and functions, invariance of sets, subdifferential sets, contingent derivatives.

AMS subject classifications. 37B25, 47J35, 49J52, 93B05.

1. Introduction. With the ever-increasing influence of mathematical modeling and engineering in various branches of applications, it is not surprising that the theory of differential inclusions has played a central role in the understanding of various applications, including for instance, physical problems, biological and physiological systems, population dynamics. The stability analysis of the different systems involved in real applications and more precisely the characterization of the evolution of a point to its stable state has been studied by many authors since the pioneering work by Lyapunov at the end of the 19th century.

The main feature of Lyapunov's approach is that it does not require an explicit expression for the solutions of the dynamic system. In order to find good candidates (energy-like) functions which well-behave with the underlying trajectories, we only need to use the involved data. In the literature such functions are termed Lyapunov's functions. This indirect method is very useful especially when dealing with complex real-world applications, where only the behavior of the system is of major interest (such as, for instance, the behavior at infinite-time or the stability of the equilibrium sets).

The aim of this paper is to provide explicit primal and dual criteria for weakly lower semicontinuous (lsc, for short) Lyapunov functions, or, more generally, for Lyapunov pairs associated to a first-order differential inclusion of the type:

$$
\dot{x}\left(t ; x_{0}\right) \in f\left(x\left(t ; x_{0}\right)\right)-A x\left(t ; x_{0}\right) \quad \text { a.e. } t \geq 0, \quad x_{0} \in \operatorname{cl}(\operatorname{Dom} A) .
$$

The resulting criteria in the approach we propose are given explicitly by means of the proximal subdifferential of the involved functions and therefore, an a priori knowledge of the semigroup generated by $-A$ is not required. Hence, this work follows the spirit of the Lyapunov indirect method since it only calls upon the known data $f$ and $A$ present in (1.1).

Here, and thereafter, $\operatorname{cl}(\operatorname{Dom} A)$ denotes the closure of the domain of a multivalued maximally monotone operator $A: H \rightrightarrows H$ defined on a real Hilbert space $H$, and $f$ is a Lips-

\footnotetext{
* Research supported by the ANR Project NT 05-1/43040, the EADS project "Solving challenging problems in automatic control", the university of Limoges, the laboratory XLIM (UMR-CNRS 6172). This paper was finished while the third author was on sabbatical. He wish to express his gratitude to Professors Marco Lopez Cerdá and Juan Parra for providing him the opportunity to stay in the universiy of Alicante and the university Miguel Hernandez in Elche.

$\dagger$ Université de Limoges, Laboratoire XLIM,123, Avenue Albert Thomas, France (samir.adly@unilim.fr).

$\ddagger$ Universidad de Chile (CMM), Avda Blanco Encalada 2120, Santiago, Chile (ahantoute@dim.uchile.cl).

$\S$ Université de Limoges, Laboratoire XLIM, 123, Avenue Albert Thomas, France (michel.thera@unilim.fr).
} 
chitz mapping defined on $\operatorname{cl}($ Dom $A$ ). A pair of proper extended-real-valued lsc functions $V, W$ : $H \rightarrow \mathbb{R} \cup\{+\infty\}$ is said to form a Lyapunov pair for the differential inclusion (1.1) if for all $x_{0} \in \operatorname{cl}(\operatorname{Dom} A)$ the solution $x\left(\cdot ; x_{0}\right)$, in the sense that will be made precise in Sect. 3, satisfies

$$
V\left(x\left(t ; x_{0}\right)\right)-V\left(x\left(s ; x_{0}\right)\right)+\int_{s}^{t} W\left(x\left(\tau ; x_{0}\right)\right) d \tau \leq 0 \quad \text { for all } t \geq s \geq 0 .
$$

Observe that for $W=0$ one recovers the classical notion of Lyapunov functions; e.g., [34]. More generally, instead of (1.2), we are going to consider functions $V, W$ satisfying, for some $a \geq 0$,

$$
e^{a t} V\left(x\left(t ; x_{0}\right)\right)-e^{a s} V\left(x\left(s ; x_{0}\right)\right)+\int_{s}^{t} W\left(x\left(\tau ; x_{0}\right)\right) d \tau \leq 0 \quad \text { for all } t \geq s \geq 0 .
$$

In this case, the (weighted) pair $(V, W)$ will be refered to as an $a$-Lyapunov pair. The main motivation in using $a$-Lyapunov pairs instead of simply functions is that many stability concepts for the equilibrium sets of (1.1), namely stability, asymptotic or finite-time stability, can be obtained just by choosing appropriate functions $W$ in (1.2). The weight $e^{a t}$ is useful for instance when exponential stability is concerned. So, even in autonomous systems like those considered in (1.1), the function $W$ or the weight $e^{a t}$ may be useful since, in some sense, they emphasize the decreasing of the Lyapunov function $V$.

Lyapunov pairs have been considered in $[14,15,17,25,30]$ among many contributions. For instance, Cârjaă \& Motreanu [15], using arguments based on tangency and flow-invariance, have given a characterization of Lyapunov pairs for a general initial value problem with a possibly multivalued m-accretive operator on an arbitrary Banach space by means of the contingent derivative related to the operator. In [14], they also have given an infinitesimal characterization of Lyapunov pairs of functions for the flow generated by a semilinear evolution equation on a Hilbert space by means of a suitable contingent derivative formulation. Kocan \& Soravia in $[24,25]$ have considered Lyapunov functions for infinite-dimensional dynamical systems governed by general maximal monotone operators. They have obtained a characterization of Lyapunov pairs by means of the associated Hamilton-Jacobi partial differential equations, whose solutions are meant in the viscosity sense. The criteria in $[14,15,24,25]$ are given by means of the semigroup generated by $-A$; see also $[8,10,16]$ for related results. In the case of a differentiable semigroup generated by $-A$, explicit characterizations for some classes of invariant sets are given in [8], by means of the tangent cones to these sets. Criteria for Lyapunov pairs with respect to the homogeneous counterpart of (1.1) (i.e., $f=0$ ) were given in [30] in term of the resolvent of $A$ (see Corollary 5.2 in Sect. 5). Using deep tools from nonsmooth analysis, Clarke et al gave in [20] sufficient criteria by means of Hamilton-Jacobi equations whose solutions are the lsc Lyapunov functions (called by these authors functions that weak or strong decrease along the trajectories). This approach applies to (1.1) when the right-hand-side (RHS) is replaced by an upper semicontinuous setvalued mapping with nonempty convex and compact values. Nonsmooth Lyapunov functions were also investigated for different models; in $[7,18,32]$ for locally Lipschitz Lyapunov functions, in [12] for convex Lyapunov functions, in $[5,6,17,25,30,37]$ for lsc Lyapunov functions, etc. In [1] and [23], smooth Lyapunov functions were used in the framework of the stability of second order differential equations and non-linear mechanical systems with frictional and unilateral constraints.

If we assume that $V$ is sufficiently smooth (of class $\mathcal{C}^{1}$ on $H$ for example), then by an elementary derivation of the composite function $t \mapsto e^{a t} V\left(x\left(t ; x_{0}\right)\right)$, it is not difficult to show 
that $(V, W)$ forms an $a$-Lyapunov pair for (1.1) if, for instance,

$$
\sup _{y \in H} \sup _{y^{*} \in A y}\left(V^{\prime}(y)\left(f(y)-y^{*}\right)+a V(y)+W(y)\right) \leq 0
$$

(with the convention $\left.\sup _{\emptyset}=-\infty\right)$. Hence, provided that $V$ and $W$ have a good behavior around the equilibrium sets of (1.1) (namely, semidefiniteness and appropriate continuity of $V$ ), one can derive many stability properties of the solution $x\left(\cdot ; x_{0}\right)$. Meanwhile, the assumption on the smoothness of $V$ reduces the efficiency of this general approach as long as complex systems are considered. Therefore, relaxing the condition on the smoothness allows more flexibility in determining such functions. Our approach is also useful especially when dealing with multiple isolated equilibrium sets for (1.1). More precisely, to study each equilibrium set separately, a candidate Lyapunov function can be chosen to have infinite value outside a neighborhood of this set. This fact is possible if one considers lsc extended real-valued candidate functions as we do in this paper. Since our goal is to provide criteria for nonsmooth (lsc) Lyapunov pairs, with respect to (1.1), instead of the derivative of $V$ in (1.3), our study will involve the usage of appropriate tools of advanced variational analysis and generalized differentiation, namely the proximal, horizontal, and the limiting subdifferentials, normal cones, Dini directional derivative, etc.

It is useful to recall that the concept of Lyapunov pairs can be equivalently written in terms of (forward) invariance. We recall that a nonempty closed set $S \subset H$ is invariant for (1.1) if the whole trajectory $x\left(\cdot ; x_{0}\right)$ is kept in $S$ whatever it starts in $S\left(x_{0} \in S \cap \operatorname{cl}(\operatorname{Dom} A)\right)$. In general, in the framework of general differential inclusions, if for every $x_{0} \in S$ one can find at least one solution starting at $x_{0}$ and which remains all the time in $S$, then $S$ is termed a viable set for this differential inclusion; there exists a wide literature on viability theory (see for instance the books by Aubin [2] and Aubin \& Cellina [3] and the references therein). In our setting, due to the uniqueness of the solution of (1.1), both concepts are equivalent. On another hand, it can be easily shown that $V$ is a Lyapunov function for (1.1) if and only if its epigraph is invariant for the (augmented in $H \times \mathbb{R}$ ) differential inclusion

$$
\dot{z}\left(t ; x_{0}, \alpha_{0}\right) \in \tilde{f}\left(z\left(t ; x_{0}, \alpha_{0}\right)\right)-\widetilde{A} z\left(t ; x_{0}, \alpha_{0}\right) \text { a.e. } t \geq 0, z\left(0, x_{0}, \alpha_{0}\right)=\left(x_{0}, \alpha_{0}\right) \in \operatorname{cl}(\operatorname{Dom} \widetilde{A}),
$$

where $\tilde{f}:=(f, 0)$ and $\widetilde{A}:=(A, 0)$ are defined on $H \times \mathbb{R}$. Conversely, a nonempty closed set $S$ is invariant for (1.1) if the indicator function of $S$ is a Lyapunov function for (1.1). In this respect, Nagumo's Theorem (e.g., $[16,36]$ and the references therein) states that a closed set $S$ is invariant for an ODE of the form

$$
\dot{x}\left(t ; x_{0}\right)=F\left(x\left(t ; x_{0}\right)\right)
$$

if and only if for each $y \in \mathbb{R}^{n}$ the vector $F(y)$ of the RHS is contingent in the sense of Bouligand to $S$ at $y$. Next, this result was adapted by Brézis [11] to non-autonomous ODE in infinitedimensional (Banach) spaces, under some supplementary Lipschitz continuity conditions of the RHS; see also [29] for related results.

The organization of the rest of the paper is as follows. In Section 2, we present briefly tools and basic results that are necessary for our approach. In Section 3 we focus on new primal and dual criteria for weakly lsc Lyapunov pairs. This is achieved in Theorem 3.3 using many dual conditions involving the subdifferential of the nominal functions. The particular case when the topological interior of the set $\operatorname{co}(\operatorname{Dom} A)$ is nonempty is addressed in Theorem 3.4. While, the primal condition involving the directional derivative is given in Theorem 3.5. The counterpart of these results for the invariance of closed subsets is established in Theorem 3.6. These abstract 
results are illustrated in Section 4 in which we exhibit some concrete examples of nonsmooth Lyapunov pairs. In Section 5, we make a review of some other criteria for Lyapunov pairs. Section 6 is devoted to complete the proofs of the main results given in Section 3.

2. Notation and main tools. In this section we introduce some fundamental definitions, properties and notations which are needed. Throughout the paper, $H$ stands for a (real) Hilbert space, endowed with the inner product $\langle\cdot, \cdot\rangle$ and associated norm $\|\cdot\|$, and identified to its topological dual $H^{\prime}$. If $H$ is a finite Cartesian product of Hilbert spaces $\left(H_{i},\|\cdot\|_{i}\right), i=1, \cdots, k$, then we use the $\ell^{1}$-norm given by $\|\cdot\|=\|\cdot\|_{1}+\cdots\|\cdot\|_{k}$. We write $\rightarrow, \rightarrow$ (or weak-lim) to denote the strong and weak convergence, respectively, and $\underset{S}{\vec{S}}, \vec{S}$ if the convergence is restricted to a set $S \subset H$. The null vector in $H$ is denoted by $\theta$. For $x \in X, B \rho(x)$ is the open ball with center $x$ and radius $\rho>0$ so that $B:=B_{1}(\theta)$ is the unit open ball. For $a, b \in \overline{\mathbb{R}}:=\mathbb{R} \cup\{+\infty,-\infty\}$, $[a, b)$ is the line segment excluding $b$, and $a^{+}:=\max \{0, a\}$. The following notations and facts are basically standard in convex, variational, and set-valued analysis; the reader can consult the books $[19,27,31]$ for more details, discussions, and further references.

Given a set $S \subset H$ (or $S \subset H \times \mathbb{R}$ ), by $\operatorname{co} S$, Int $S$, and $\operatorname{cl} S$ (or $\bar{S}$ ), we denote the convex hull, the interior, and the closure of the set $S$, respectively. The indicator function of $S$ and the distance function to $S$ are given, respectively, by

$$
\delta_{S}(x):=0 \text { if } x \in S ;+\infty \text { if not, } d(x, S):=\inf \{\|x-y\|: y \in S\} .
$$

The orthogonal projection mapping, $\Pi_{S}$, onto $S$ is defined as

$$
\Pi_{S}(x):=\{y \in S:\|x-y\|=d(x, S)\}
$$

For a multivalued operator $A: H \rightrightarrows H$, the domain and the graph are given, respectively, by

$$
\operatorname{Dom} A:=\{x \in H \mid A x \neq \emptyset\}, \operatorname{gph} A:=\{(x, y) \in H \times H \mid y \in A x\} ;
$$

it is convenient to identify an operator $A$ to its graph in $H \times H$. The inverse operator of $A$ is $A^{-1}: H \rightrightarrows H$ defined as

$$
(y, x) \in A^{-1} \Longleftrightarrow(x, y) \in A .
$$

For $\lambda>0$, the resolvent and the Yoshida approximation of $A$ are given, respectively, by

$$
J_{\lambda}:=(I+\lambda A)^{-1}, A_{\lambda}:=\frac{1}{\lambda}\left(I-J_{\lambda}\right),
$$

where $I: H \rightrightarrows H$ stands for the identity mapping. The operator $A$ is said to be monotone if

$$
\left\langle y_{1}-y_{2}, x_{1}-x_{2}\right\rangle \geq 0 \text { for all }\left(x_{i}, y_{i}\right) \in A, i=1,2,
$$

and maximally monotone if $A$ is not properly included in any other monotone operator. If $A$ is maximally monotone, it is well known (e.g., Simons [33]) that cl(Dom $A$ ) is convex, and the set $A x$ is convex and closed for every $x \in H$. We recall that, for every $\lambda>0, J_{\lambda}$ is defined everywhere, single-valued, nonexpansive, and satisfies $A_{\lambda} x \in A\left(J_{\lambda} x\right)$ and $\lim _{\lambda \rightarrow 0^{+}} J_{\lambda} x=x$ for every $x \in$ $\operatorname{cl}(\operatorname{Dom} A)$. If Int $(\operatorname{co}\{\operatorname{Dom} A\}) \neq \emptyset$, then $\operatorname{Int}(\operatorname{Dom} A)=\operatorname{Int}(\operatorname{cl}(\operatorname{Dom} A))=\operatorname{Int}(\operatorname{co}\{\operatorname{Dom} A\})$, and $A$ is locally bounded on Int (Dom $A$ ). Moreover, if $(A x)^{\circ}$ denotes the set of points of minimal norm in $A(x)$, i.e., $(A x)^{\circ}:=\left\{y \in A x:\|y\|=\min _{z \in A x}\|z\|\right\}$, then, for every $x \in$ Dom $A$ we have 
that $\lim _{\lambda \rightarrow 0^{+}} A_{\lambda} x=(A x)^{\circ}=\Pi_{A x}(\theta)$, or, more generally,

$$
\lim _{\lambda \rightarrow 0^{+}} A_{\lambda}(x+\lambda y)=\Pi_{A x}(y) \text { for every } y \in H .
$$

In particular, if $A=\partial h$ is the subdifferential in the sense of convex analysis of some lsc convex proper function $h: H \rightarrow \overline{\mathbb{R}}$ (see (2.4)), then $A$ is typically an example of a maximally monotone operator (however, not all maximally monotone operators are convex subdifferentials) and we know that

$\operatorname{Dom} A \subset \operatorname{Dom} h \subset \operatorname{cl}(\operatorname{Dom} h)=\operatorname{cl}(\operatorname{Dom} A)$ by

Given a function $\varphi: H \rightarrow \overline{\mathbb{R}}$, its (effective) domain and epigraph are defined, respectively,

$$
\operatorname{Dom} \varphi:=\{x \in H \mid \varphi(x)<+\infty\}, \text { epi } \varphi:=\{(x, \alpha) \in H \times \mathbb{R} \mid \varphi(x) \leq \alpha\} .
$$

For $\lambda \in \overline{\mathbb{R}}$, the set $[\varphi>\lambda]:=\{x \in H \mid \varphi(x)>\lambda\}$ signifies the open upper-level set of $\varphi$ at height $\lambda$. We use the notations

$$
\mathcal{F}(H):=\{\varphi: H \rightarrow \overline{\mathbb{R}} \mid \varphi \text { proper and lsc }\}, \mathcal{F}_{w}(H):=\{\varphi: H \rightarrow \overline{\mathbb{R}} \mid \varphi \text { proper and weakly lsc }\},
$$

and denote by $\mathcal{F}^{+}(H)$ and $\mathcal{F}_{w}^{+}(H)$ the subsets of those functions, respectively in $\mathcal{F}(H)$ and $\mathcal{F}_{w}(H)$ which are nonnegative. If $\varphi \in \mathcal{F}(H), x \in \operatorname{Dom} \varphi$, and $\xi \in H$. We call $\xi$ a proximal subgradient of $\varphi$ at $x$, written $\xi \in \partial_{P} \varphi(x)$, if there are $\rho>0$ and $\sigma \geq 0$ such that

$$
\varphi(y) \geq \varphi(x)+\langle\xi, y-x\rangle-\sigma\|y-x\|^{2} \text { for all } y \in B_{\rho}(x) .
$$

We recall that $\partial_{P} \varphi(x)$ is convex, possibly empty, and not necessarily closed. More generally, $\xi$ is said a Fréchet subgradient of $\varphi$ at $x$, written $\xi \in \partial_{F} \varphi(x)$, if

$$
\varphi(y) \geq \varphi(x)+\langle\xi, y-x\rangle+o(\|y-x\|) \text { for all } y \in H .
$$

Associated to these concepts, limiting objects have been introduced: we call $\xi$ a limiting subdifferential of $\varphi$ at $x$, written $\xi \in \partial_{L} \varphi(x)$, if there exist sequences $\left(x_{k}\right)_{k},\left(\xi_{k}\right)_{k} \subset H$ such that

$$
\left.x_{k} \underset{\varphi}{\rightarrow} x \text { (i.e., } x_{k} \rightarrow x, \varphi\left(x_{k}\right) \rightarrow \varphi(x)\right), \xi_{k} \in \partial_{P} \varphi\left(x_{k}\right), \xi_{k} \rightarrow \xi
$$

while, $\xi$ is a horizontal subgradient of $\varphi$ at $x$, written $\xi \in \partial_{\infty} \varphi(x)$, if there exist sequences $\left(\alpha_{k}\right)_{k} \subset \mathbb{R}_{+}$and $\left(x_{k}\right)_{k},\left(\xi_{k}\right)_{k} \subset H$ such that

$$
\alpha_{k} \rightarrow 0^{+}, x_{k} \underset{\varphi}{\rightarrow} x, \xi_{k} \in \partial_{P} \varphi\left(x_{k}\right), \alpha_{k} \xi_{k} \rightarrow \xi
$$

For comparative purposes, we recall that in the present setting the Clarke subdifferential of $\varphi$ at $x$ is defined by the so-called representation formula (see, e.g., [28])

$$
\partial_{C} \varphi(x)=\overline{\operatorname{co}}\left\{\partial_{L} \varphi(x)+\partial_{\infty} \varphi(x)\right\}
$$

from which it clearly follows that $\partial_{P} \varphi(x) \subset \partial_{F} \varphi(x) \subset \partial_{L} \varphi(x) \subset \partial_{C} \varphi(x)$. In particular, if $\varphi$ is Gâteaux-differentiable at $x$ and $\varphi_{G}^{\prime}(x)$ denotes its Gâteaux-differential, then $\partial_{P} \varphi(x) \subset$ $\left\{\varphi_{G}^{\prime}(x)\right\} \subset \partial_{C} \varphi(x)$. If $\varphi$ is $\mathcal{C}^{1}$, then $\partial_{P} \varphi(x) \subset\left\{\varphi^{\prime}(x)\right\}=\partial_{C} \varphi(x)$ and $\partial_{\infty} \varphi(x)=\{\theta\}$. If $\varphi$ is $\mathcal{C}^{2}$, then $\partial_{P} \varphi(x)=\partial_{C} \varphi(x)=\left\{\varphi^{\prime}(x)\right\}$. If $\varphi$ is convex, then all these subdifferential concepts (except 
the horizontal) coincide with the usual Fenchel-Moreau-Rockafellar subdifferential set

$$
\partial \varphi(x):=\{y \in H \mid \varphi(z)-\varphi(x) \geq\langle y, z-x\rangle \text { for all } z \in H\} .
$$

Finally, let us mention that if $x \notin \operatorname{Dom} \varphi$, then by convention we set $\partial_{P} \varphi(x)=\partial_{F} \varphi(x)=$ $\partial_{L} \varphi(x)=\partial_{C} \varphi(x)=\emptyset$.

From a geometrical point of view, if $S \subset H$ is a closed set and $x \in S$, the proximal normal cone to $S$ at $x$ is $\mathrm{N}_{S}^{P}(x):=\partial_{P} \delta_{S}(x)$ or, equivalently,

$$
\mathrm{N}_{S}^{P}(x)=\operatorname{cone}\left(\Pi_{S}^{-1}(x)-x\right) \text { if } \Pi_{S}^{-1}(x) \neq \emptyset ;\{\theta\} \text { if } \Pi_{S}^{-1}(x)=\emptyset .
$$

We shall also use $\widetilde{\mathrm{N}}_{S}^{P}(x)$ to denote the subset of $\mathrm{N}_{S}^{P}(x)$ given by

$$
\widetilde{\mathrm{N}}_{S}^{P}(x):=\left\{y \in H:\langle y, z-x\rangle \leq\|z-x\|^{2} \text { for all } z \in S \text { closed to } x\right\} .
$$

Similarly, $\mathrm{N}_{S}^{L}(x):=\partial_{L} \delta_{S}(x)\left(=\partial_{\infty} \delta_{S}(x)\right)$ is the limiting normal cone to $S$ at $x$, and $\mathrm{N}_{S}^{C}(x):=$ $\overline{\mathrm{co}}\left\{\mathrm{N}_{S}^{L}(x)\right\}$ is the Clarke normal cone to $S$ at $x$. We call contingent cone to $S$ at $x \in S$ (or Bouligand tangent cone), written $\mathrm{T}_{S}(x)$, the set

$$
\mathrm{T}_{S}(x):=\left\{y \in H \mid x+\tau_{k} y_{k} \in S \text { for all } k \text {, for some } y_{k} \rightarrow y \text { and } \tau_{k} \rightarrow 0^{+}\right\} .
$$

Moreover, we have that $\partial_{P} \varphi(x) \times\{-1\}=\mathrm{N}_{\text {epi } \varphi}^{P}(x, \varphi(x)) \cap(H \times\{-1\}), \partial_{\infty} \varphi(x) \times\{0\}=$ $\mathrm{N}_{\text {epi } \varphi}^{P}(x, \varphi(x)) \cap(H \times\{0\})$, and $\mathrm{T}_{\text {epi } \varphi}(x, \varphi(x))=\operatorname{epi} \varphi^{\prime}(x, \cdot)$, where $\varphi^{\prime}(x, \cdot): H \rightarrow \overline{\mathbb{R}}$ denotes the Dini directional derivative of $\varphi$ at $x$, given by

$$
\varphi^{\prime}(x, v):=\liminf _{t \rightarrow 0^{+}, w \rightarrow v} \frac{\varphi(x+t w)-\varphi(x)}{t} ;
$$

hence, if $\varphi$ is convex, then $\varphi^{\prime}(x, v)=\inf _{t>0} t^{-1}(\varphi(x+t v)-\varphi(x))$ is the usual directional derivative.

3. A general criterion for nonsmooth Lyapunov pairs. In this section, $H$ is a Hilbert space, and $A: H \rightrightarrows H, f: \operatorname{cl}(\operatorname{Dom} A) \subset H \rightarrow H$ denote a maximally monotone operator and a $L_{f}$-Lipschitz continuous mapping, respectively. Our aim is to provide explicit characterizations of weakly lower semicontinuous Lyapunov pairs with respect to the differential inclusion (1.1):

$$
\dot{x}\left(t ; x_{0}\right) \in f\left(x\left(t ; x_{0}\right)\right)-A x\left(t ; x_{0}\right) \quad \text { a.e. } t \geq 0, x_{0} \in \operatorname{cl}(\operatorname{Dom} A) .
$$

Let us first precise the sense in which we are considering solutions of (1.1); the reader is refered to [12] for more details. Fix $T>0$ and $x_{0} \in \operatorname{cl}(\operatorname{Dom} A)$. By a strong solution we mean a function $x\left(\cdot ; x_{0}\right):[0, T] \rightarrow H$ defined and continuous on $[0, T]$, verifying the initial condition $x\left(0 ; x_{0}\right)=x_{0}$, absolutely continuous on every interval $[a, b] \subset(0, T)$, and satisfying

$$
x\left(t ; x_{0}\right) \in \operatorname{Dom} A, \dot{x}\left(t ; x_{0}\right) \in f\left(x\left(t ; x_{0}\right)\right)-A x\left(t ; x_{0}\right) \quad \text { a.e. } t \in(0, T) .
$$

If such a solution exists it must be unique. Further, we have that $\dot{x}\left(\cdot ; x_{0}\right) \in L^{\infty}([0, T), H)$ if and only if $x_{0} \in \operatorname{Dom} A$. In this later case, for all $t \in[0, T), x\left(t ; x_{0}\right) \in \operatorname{Dom} A$ and $x\left(\cdot ; x_{0}\right)$ is right-derivable at $t$ with

$$
\left.\frac{d^{+}}{d t} x\left(t ; x_{0}\right)=\left(f\left(x\left(t ; x_{0}\right)\right)-A x\left(t ; x_{0}\right)\right)\right)^{\circ}=f\left(x\left(t ; x_{0}\right)\right)-\Pi_{A x\left(t ; x_{0}\right)}\left(f\left(x\left(t ; x_{0}\right)\right)\right) .
$$

For instance, a strong solution exists in each of the following cases (see Brézis [12]): $x_{0} \in \operatorname{Dom} A$; 
Int $(\operatorname{co}(\operatorname{Dom} A)) \neq \emptyset ; \operatorname{dim} H<\infty ; A=\partial \varphi$ for some convex function $\varphi \in \mathcal{F}(H)$; etc. In the general case, it is well established (see e.g., [9]) that Equation (1.1) admits a unique weak solution $x\left(\cdot ; x_{0}\right) \in \mathcal{C}(0, T ; H)$ which satisfies $x\left(t ; x_{0}\right) \in \operatorname{cl}($ Dom $A)$ for all $t \geq 0$. Equivalently, there exists a sequence $\left(z_{k}\right)_{k \in \mathbb{N}} \subset$ Dom $A$ converging to $x_{0}$ such that the strong solution $x_{k}\left(\cdot ; z_{k}\right)$ of the equation

$$
\dot{x}_{k}\left(t ; z_{k}\right) \in f\left(x_{k}\left(t ; z_{k}\right)\right)-A x_{k}\left(t ; z_{k}\right) \quad \text { a.e. } t \geq 0, x_{k}\left(0, z_{k}\right)=z_{k}
$$

uniformly converges to $x\left(\cdot ; x_{0}\right)$ on $[0, T]$. Moreover, we have that

$$
\begin{gathered}
x\left(s ; x\left(t ; x_{0}\right)\right)=x\left(s+t ; x_{0}\right) \text { for all } s, t \geq 0, \\
\left\|x\left(t ; x_{0}\right)-x\left(t ; y_{0}\right)\right\| \leq e^{L_{f} t}\left\|x_{0}-y_{0}\right\| \text { for all } t \geq 0, x_{0}, y_{0} \in \operatorname{cl}(\operatorname{Dom} A) .
\end{gathered}
$$

In the remainder of the paper, $x\left(\cdot ; x_{0}\right)$ will denote the weak (strong, if applicable) solution of Equation (1.1).

Definition 1. Let $V \in \mathcal{F}(H), W \in \mathcal{F}(H)$, and $a \in \mathbb{R}_{+}$be given. We say that $(V, W)$ forms an a-Lyapunov pair for (1.1) if for all $x_{0} \in \operatorname{cl}(\operatorname{Dom} A)$ we have that

$$
e^{a t} V\left(x\left(t ; x_{0}\right)\right)+\int_{0}^{t} W\left(x\left(\tau ; x_{0}\right)\right) d \tau \leq V\left(x_{0}\right) \text { for all } t \geq 0 .
$$

In particular, if $a=0$, we call $(V, W)$ a Lyapunov pair and, in addition, if $W=0$ then $V$ is said a Lyapunov function. The last inequality above is refered to as the Lyapunov inequality associated to (1.1).

As we observe in the following lemma, for which a proof can be found in Clarke et al [21], the lack of regularity of Lyapunov pairs is mainly due to the non-smoothness of the first component function.

LEMMA 3.1. If $W \in \mathcal{F}^{+}(H)$, then there exists a sequence of functions $\left(W_{k}\right)_{k \in \mathbb{N}} \subset \mathcal{F}^{+}(H)$ converging pointwisely to $W$ (for instance, $W_{k} \nearrow W$ ) such that $W_{k}$ is Lipschitz continuous on every bounded subset of $H$. Consequently, for each $k$ and $y \in H$, we have that $W(y)>0$ if and only if $W_{k}(y)>0$. Moreover, for given $V \in \mathcal{F}^{+}(H)$ and $a \geq 0,(V, W)$ forms an a-Lyapunov pair for (1.1) if and only if each $\left(V, W_{k}\right)$ is an a-Lyapunov pair.

It is also worth recalling that the very essential point in Lyapunov's theory is that a Lyapunov function decreases along the trajectories of (1.1). Our definition also reflects this effect according to the following proposition.

Proposition 3.2. Let $V \in \mathcal{F}_{w}(H), W \in \mathcal{F}^{+}(H)$, and $a \in \mathbb{R}_{+}$be given. Then, the following are equivalent:

(i) $(V, W)$ forms an a-Lyapunov pair for (1.1);

(ii) for every $x_{0} \in \operatorname{cl}(\operatorname{Dom} A)$ we have that

$$
e^{a t} V\left(x\left(t ; x_{0}\right)\right)-e^{a s} V\left(x\left(s ; x_{0}\right)\right)+\int_{s}^{t} W\left(x\left(\tau ; x_{0}\right)\right) d \tau \leq 0 \text { for all } t, s \geq 0 ;
$$


(iii) for every $x_{0} \in \operatorname{cl}(\operatorname{Dom} A)$ there is some $\delta>0$ such that

$$
e^{a t} V\left(x\left(t ; x_{0}\right)\right)+\int_{0}^{t} W\left(x\left(\tau ; x_{0}\right)\right) d \tau \leq V\left(x_{0}\right) \text { for all } t \in[0, \delta] .
$$

Proof. Implication (ii) $\Longrightarrow$ (iii) is immediate. To establish (i) $\Longrightarrow$ (ii), without loss of generality (w.l.o.g., for short), by Lemma 3.1 we might assume that $W$ is continuous (which makes the integrals easier to handle). Then, if $x_{0} \in \operatorname{cl}(\operatorname{Dom} A)$ is fixed, combining Definition 1 with (3.3) it follows that, for all $t \geq s \geq 0$,

$$
\begin{aligned}
& e^{a(s+t)} V\left(x\left(s+t ; x_{0}\right)\right)+\int_{0}^{s+t} W\left(x\left(\tau ; x_{0}\right)\right) d \tau \\
& =e^{a t} e^{a s} V\left(x\left(s ; x\left(t ; x_{0}\right)\right)\right)+\int_{0}^{t} W\left(x\left(\tau ; x_{0}\right)\right) d \tau+\int_{0}^{s} W\left(x\left(\tau ; x\left(t ; x_{0}\right)\right)\right) d \tau \\
& \leq e^{a t} V\left(x\left(t ; x_{0}\right)\right)-e^{a t} \int_{0}^{s} W\left(x\left(\tau ; x\left(t ; x_{0}\right)\right)\right) d \tau+\int_{0}^{t} W\left(x\left(\tau ; x_{0}\right)\right) d \tau \\
& \quad+\int_{0}^{s} W\left(x\left(\tau ; x\left(t ; x_{0}\right)\right)\right) d \tau \leq e^{a t} V\left(x\left(t ; x_{0}\right)\right)+\int_{0}^{t} W\left(x\left(\tau ; x_{0}\right)\right) d \tau ;
\end{aligned}
$$

that is, (ii) holds.

To show that (iii) $\Longrightarrow\left(\right.$ i) holds, we fix $x_{0} \in \operatorname{cl}(\operatorname{Dom} A)$ and set

$$
\bar{\delta}:=\sup \left\{\delta \geq 0 \mid e^{a t} V\left(x\left(t ; x_{0}\right)\right)+\int_{0}^{t} W\left(x\left(\tau ; x_{0}\right)\right) d \tau \leq V\left(x_{0}\right) \text { for all } t \in[0, \delta]\right\} .
$$

Taking into account the current assumption (iii), we observe that $\bar{\delta}>0$. Proceeding by contradiction, we suppose that $\bar{\delta}<\infty$, and using the semi-continuity of $V$ we derive that

$$
e^{a \bar{\delta}} V\left(x\left(\bar{\delta} ; x_{0}\right)\right)+\int_{0}^{\bar{\delta}} W\left(x\left(\tau ; x_{0}\right)\right) d \tau \leq V\left(x_{0}\right) .
$$

But $x\left(\bar{\delta} ; x_{0}\right) \in \operatorname{cl}(\operatorname{Dom} A)$, and so by (iii) there exists some $\varepsilon>0$ such that

$$
e^{a t} V\left(x\left(t ; x\left(\bar{\delta} ; x_{0}\right)\right)+\int_{0}^{t} W\left(x\left(\tau ; x\left(\bar{\delta} ; x_{0}\right)\right)\right) d \tau \leq V\left(x\left(\bar{\delta} ; x_{0}\right)\right) \text { for all } t \in(0, \varepsilon] .\right.
$$

Hence, using again (3.3), for every $t \in(0, \varepsilon]$, we obtain

$$
\begin{aligned}
& e^{a(t+\bar{\delta})} V\left(x\left(t+\bar{\delta} ; x_{0}\right)\right)+\int_{0}^{t+\bar{\delta}} W\left(x\left(\tau ; x_{0}\right)\right) d \tau \\
& =e^{a \bar{\delta}} e^{a t} V\left(x\left(t ; x\left(\bar{\delta} ; x_{0}\right)\right)+\int_{0}^{\bar{\delta}} W\left(x\left(\tau ; x_{0}\right)\right) d \tau+\int_{0}^{t} W\left(x\left(\tau ; x\left(\bar{\delta} ; x_{0}\right)\right)\right) d \tau\right. \\
& \leq e^{a \bar{\delta}}\left(V\left(x\left(\bar{\delta} ; x_{0}\right)\right)+\int_{0}^{\bar{\delta}} W\left(x\left(\tau ; x_{0}\right)\right) d \tau\right. \\
& \leq V\left(x_{0}\right),
\end{aligned}
$$

and the contradiction $\bar{\delta}<\varepsilon+\bar{\delta} \leq \bar{\delta}$.

The next example shows the necessity of considering nonsmooth Lyapunov's pairs or functions; it will be continued in Example 4.4. 
EXAMPLE 3.1. We consider the one-dimensional systems with the parameter $\lambda \in \mathbb{R}_{+}$,

$$
\dot{x}_{\lambda}\left(t ; x_{0}\right) \in-\operatorname{Sign}\left(x_{\lambda}\left(t ; x_{0}\right)\right)+\lambda\left|x_{\lambda}\left(t ; x_{0}\right)\right| \quad \text { a.e. } t \geq 0, x_{\lambda}\left(0 ; x_{0}\right)=x_{0} \in \mathbb{R}
$$

where $\operatorname{Sign}(\alpha):=1$ if $\alpha>0 ;-1$ if $\alpha<0 ;[-1,1]$ if $\alpha=0$. These systems can be reformulated in the form (1.1) by taking $A=\partial|\cdot|$ and $f_{\lambda}=\lambda|\cdot|$.

If $\lambda=0$, then 0 is the unique equilibrium point and the solution is given by

$$
x_{0}\left(t ; x_{0}\right):= \begin{cases}\max \left\{0, x_{0}-t\right\} & \text { if } x_{0} \geq 0 \\ \min \left\{0, x_{0}+t\right\} & \text { if } x_{0} \leq 0\end{cases}
$$

In view of $(1.3)$, the smooth function $V_{0}(x):=\frac{1}{2} x^{2}$ is a Lyapunov function since that

$$
\left\langle\nabla V_{0}(x),-\operatorname{Sign} x\right\rangle=-|x| \leq 0 \text { for all } x \in \mathbb{R}
$$

It follows that the function $V(x)=|x|, x \in \mathbb{R}$, is also a Lyapunov function for (3.4), but this fact could not be checked using (1.3) since $|\cdot|$ fails to be differentiable at 0 .

If $\lambda>0$, the equilibrium points are $\left\{0, \frac{1}{\lambda}\right\}$ and the solution is given by

$$
x_{\lambda}\left(t ; x_{0}\right):= \begin{cases}\max \left\{0, e^{\lambda t}\left(x_{0}-\frac{1}{\lambda}\right)+\frac{1}{\lambda}\right\} & \text { if } x_{0} \geq 0 \\ \min \left\{0, e^{-\lambda t}\left(x_{0}-\frac{1}{\lambda}\right)+\frac{1}{\lambda}\right\} & \text { if } x_{0} \leq 0\end{cases}
$$

If we consider again the smooth function $V_{0}(x)=\frac{1}{2} x^{2}$, now (1.3) is written

$$
\left\langle\nabla V_{0}(x),-\operatorname{Sign} x+\lambda|x|\right\rangle=|x|(\lambda x-1),
$$

so that an inequality as in (3.5) is valid only for $x \leq \frac{1}{\lambda}$. Consequently, an expected Lyapunov function would be the lsc extended real-valued function

$$
V_{\lambda}(x)=\frac{1}{2} x^{2} \text { if } x \leq \frac{1}{\lambda} \text { and } V_{\lambda}(x)=+\infty \text { otherwise. }
$$

The following theorem gives the desired characterization of weakly lower semicontinuous Lyapunov's pairs with respect to (1.1). More generally, we establish a general principle for the Lyapunov inequality to hold locally on upper-level sets of the candidate function $V$.

We recall that $\mathcal{F}_{w}(H)$ (and $\left.\mathcal{F}^{+}(H)\right)$ and $\widetilde{\mathrm{N}}_{\text {epi } V}^{P}(y, V(y)$ ) are defined in (2.2) and (2.5), respectively, and that by convention $\sup _{\emptyset}=-\infty$. The proof of the part $(\mathrm{v}) \Longrightarrow$ (i) will be completed in Section 6.

TheOrem 3.3. We let $V \in \mathcal{F}_{w}(H), W \in \mathcal{F}^{+}(H), a \in \mathbb{R}_{+}$be given, and fix $\bar{y} \in \operatorname{cl}(\operatorname{Dom} A)$, $\bar{\lambda} \in[-\infty, V(\bar{y}))$, and $\bar{\rho} \in(0,+\infty]$ so that

$$
B_{\bar{\rho}}(\bar{y}) \cap[V>\bar{\lambda}] \cap \operatorname{Dom} V \subset \operatorname{cl}(\operatorname{Dom} A) .
$$

Then, the following statements are equivalent:

(i) $\forall x_{0} \in B_{\bar{\rho}}(\bar{y}) \cap[V>\bar{\lambda}] \cap \operatorname{Dom} V$,

$$
e^{a t} V\left(x\left(t ; x_{0}\right)\right)+\int_{0}^{t} W\left(x\left(\tau ; x_{0}\right)\right) d \tau \leq V\left(x_{0}\right) \quad \forall t \in\left[0, \rho\left(x_{0}\right)\right],
$$


where $\rho\left(x_{0}\right)$ is the positive (possibly $+\infty$ ) number given by

$\rho\left(x_{0}\right)=\sup \left\{\begin{array}{l|l}\nu>0 & \begin{array}{l}\exists \rho>0 \text { with } B_{\rho}\left(x_{0}\right) \subset B_{\bar{\rho}}(\bar{y}) \cap[V>\bar{\lambda}] \text { s.t. } \forall s \in[0, \nu]: \\ \left.2\left\|x\left(s ; x_{0}\right)-x_{0}\right\|<\rho \text { and }\left|\left(e^{-a s}-1\right) V\left(x_{0}\right)-\int_{0}^{s} W\left(x\left(\tau ; x_{0}\right)\right) d \tau\right|\right\}<\rho\end{array}\end{array} ; ;\right.$

(ii) $\forall y \in B_{\bar{\rho}}(\bar{y}) \cap[V>\bar{\lambda}]$ and $\delta>0$,

$$
\begin{gathered}
\sup _{\xi \in \partial_{P} V(y)} \liminf _{\substack{\text { Dom } A \\
\rightarrow}}\left\langle\xi+\delta(y-z), f(z)-\Pi_{A z}(f(z))\right\rangle+a V(y)+W(y) \leq 0, \\
\sup _{\xi \in \partial_{\infty} V(y)} \liminf _{\substack{\operatorname{Dom} A}}\left\langle\xi+y-z, f(z)-\Pi_{A z}(f(z))\right\rangle \leq 0 ;
\end{gathered}
$$

(iii) $\forall y \in B_{\bar{\rho}}(\bar{y}) \cap[V>\bar{\lambda}]$ and $\delta>0$,

$$
\begin{aligned}
& \sup _{\xi \in \partial_{P} V(y)} \liminf _{z \rightarrow \underset{D o m}{\rightarrow} y} \lim _{\lambda \rightarrow 0^{+}}\left\langle\xi+\delta(y-z), f(y)-A_{\lambda}(z+\lambda f(z))\right\rangle+a V(y)+W(y) \leq 0, \\
& \sup _{\xi \in \partial_{\infty} V(y)} \liminf _{z \rightarrow \underset{\text { Dom }}{\rightarrow} y} \lim _{\lambda \rightarrow 0^{+}}\left\langle\xi+y-z, f(y)-A_{\lambda}(z+\lambda f(z))\right\rangle \leq 0
\end{aligned}
$$

(iv) $\forall y \in B_{\bar{\rho}}(\bar{y}) \cap[V>\bar{\lambda}]$ and $\delta>0$,

$$
\begin{aligned}
& \sup _{\xi \in \partial_{P} V(y)} \liminf _{z \rightarrow \operatorname{Dom}_{A} y} \inf _{z^{*} \in A z}\left\langle\xi+\delta(y-z), f(z)-z^{*}\right\rangle+a V(y)+W(y) \leq 0, \\
& \sup _{\xi \in \partial_{\infty} V(y)} \liminf _{z \rightarrow \underset{D o m}{\rightarrow} y} \inf _{z^{*} \in A z}\left\langle\xi+y-z, f(z)-z^{*}\right\rangle \leq 0
\end{aligned}
$$

(v) $\forall y \in B_{\bar{\rho}}(\bar{y}) \cap[V>\bar{\lambda}]$ and $(\xi, \eta) \in \widetilde{\mathrm{N}}_{\text {epi } V}^{P}(y, V(y))$,

$$
\liminf _{\substack{\mathrm{Dom} A \\ A}} \inf _{z^{*} \in A z}\left\langle\xi+y-z, f(z)-z^{*}\right\rangle \leq \eta(a V(y)+W(y)) .
$$

(vi) If $\bar{\rho}=-\bar{\lambda}=+\infty,(V, W)$ forms an a-Lyapunov pair for (1.1).

Proof. The proof of the (main) statement (v) $\Longrightarrow(\mathrm{i})$ is postponed to Section 6 . According to Lemma 3.1, we may assume first that $W$ is Lipschitz continuous on bounded subsets of $H$. Next, for each $x_{0} \in B_{\bar{\rho}}(\bar{y}) \cap[V>\bar{\lambda}] \cap$ Dom $V$, in view of the lsc of $V$ and the continuity of $x\left(\cdot ; x_{0}\right)$, we have that $\rho\left(x_{0}\right)>0$ or $\rho\left(x_{0}\right)=+\infty$. Thus, by Proposition 3.2, the last statement (vi) is equivalent to (i). The proof of (ii) $\Longrightarrow$ (iv) $\Longrightarrow(\mathrm{v})$ is immediate and the equivalence (ii) $\Longleftrightarrow$ (iii) follows from (2.1).

We are going to establish the statement (i) $\Longrightarrow$ (ii): Let us fix $y \in B_{\bar{\rho}}(\bar{y}) \cap[V>\bar{\lambda}]$, $\xi \in \partial_{P} V(y) \cup \partial_{\infty} V(y), \delta>0$, and denote

$$
\kappa(\xi):=1 \text { if } \xi \in \partial_{P} V(y) ; 0 \text { if } \xi \in \partial_{\infty} V(y) \text {; }
$$

hence, $y \in B_{\bar{\rho}}(\bar{y}) \cap[V>\bar{\lambda}] \cap \operatorname{Dom} V \subset \operatorname{cl}(\operatorname{Dom} A)$ and $(\xi,-\kappa(\xi)) \in \mathrm{N}_{\text {epi } V}^{P}(y, V(y))$. We also fix $\varepsilon>0$, and let $\left(y_{k}\right)_{k \in \mathbb{N}} \subset \operatorname{Dom} A$ be such that $y_{k} \rightarrow y$, and denote by $x_{k}\left(\cdot ; y_{k}\right)$ the (strong) 
solution of the equation

$$
\dot{x}_{k}\left(t ; y_{k}\right) \in f\left(x_{k}\left(t ; y_{k}\right)\right)-A x_{k}\left(t ; y_{k}\right) \text { a.e. } t \geq 0, \quad x_{k}\left(0, y_{k}\right)=y_{k} .
$$

By (i), and recalling that $\rho(y)>0$, we let $T \in(0, \rho(y))$ be such that

$$
\left(x(t ; y), e^{-a t} V(y)-e^{-a t} \int_{0}^{t} W(x(\tau ; y)) d \tau\right) \in \operatorname{epi} V \quad \forall t \in[0, T] .
$$

Let us introduce the mapping $h:[0, T] \subset \mathbb{R} \rightarrow \mathbb{R}$ given by

$$
h(t):=\kappa(\xi)\left(\left(e^{-a t}-1\right) V(y)-e^{-a t} \int_{0}^{t} W(x(\tau ; y)) d \tau\right) .
$$

Therefore, by the definition of $\mathrm{N}_{\text {epi } V}^{P}(y, V(y))$, there exists $\alpha>0$ such that for all $t \in[0, T]$ (w.l.o.g.)

$$
-\frac{\varepsilon}{2} \leq-\alpha\|x(t ; y)-y\|^{2}-\alpha h(t)^{2} \leq h(t)+\langle\xi, y-x(t ; y)\rangle
$$

Next using the facts that $\left(x_{k}\left(\cdot ; y_{k}\right)\right)_{k \in \mathbb{N}}$ uniformly converges to $x(\cdot ; y)$ on $[0, T]$ and $x(\cdot ; y)$ is continuous, we may also suppose that, for all $t \in[0, T]$ and all $k$,

$$
\frac{\delta}{2}\left\|y_{k}-y\right\|^{2}-\varepsilon<\left\langle\xi, y_{k}-x_{k}\left(t ; y_{k}\right)\right\rangle+h(t)+\frac{\delta}{2}\left\|x_{k}\left(t ; y_{k}\right)-y\right\|^{2}=: \varphi(t) .
$$

Then, for each $k$, we apply the Ekeland variational principle ( $[22])$, on the interval $\left[0, k^{-1} T\right]$, to find $s_{k} \in\left[0,(2 k)^{-1} T\right]$ such that $\frac{d^{+}}{d t} \varphi\left(s_{k}\right) \geq-2 \varepsilon$ (we recall that $x_{k}\left(\cdot ; y_{k}\right)$ is right-derivable at $s_{k}$ and so is $\varphi$ ). Therefore, by invoking (3.1) together with the fact that $\lim _{k \rightarrow+\infty} h^{\prime}\left(s_{k}\right)=$ $-\kappa(\xi)(a V(y)+W(y))$, for $k$ large enough we get that

$$
\left\langle\xi+\delta\left(y-x_{k}\left(s_{k} ; y_{k}\right)\right),\left(f\left(x_{k}\left(s_{k} ; y_{k}\right)\right)-A x_{k}\left(s_{k} ; y_{k}\right)\right)^{\circ}\right\rangle \leq-\kappa(\xi)(a V(y)+W(y))+2 \varepsilon .
$$

Finally, using once again the continuity of $x(\cdot ; y)$ and the uniform convergence of $x_{k}\left(\cdot ; y_{k}\right)$ to $x(\cdot ; y)$ on $[0, T]$, we obtain the convergence of $x_{k}\left(s_{k} ; y_{k}\right)$ to $y$. This completes the proof of (ii) since $\varepsilon$ is arbitrarily chosen.

Let us give some preliminary remarks on Theorem 3.3.

REMARK 3.1. (a) The use of proximal subdifferentials provides sharper conditions since it is generally strictly smaller than the Fréchet or the Clarke subdifferential (e.g., [31]). The weak lower semi-continuity of $V$ is only needed for the implication (v) $\Longrightarrow$ (i).

(b) If $V \geq 0$, statement (i) of Theorem 3.3 can be also rewritten as: for all $x_{0} \in B_{\bar{\rho}}(\bar{y}) \cap[V>$ $\bar{\lambda}] \cap \operatorname{Dom} V$,

$$
V\left(x\left(t ; x_{0}\right)\right)+\int_{0}^{t}\left[a V\left(x\left(\tau ; x_{0}\right)\right)+W\left(x\left(\tau ; x_{0}\right)\right)\right] d \tau \leq V\left(x_{0}\right) \quad \text { for all } t \in\left[0, \rho\left(x_{0}\right)\right] .
$$

(c) Statement (iv) in Theorem 3.3 holds if, for instance, $\forall y \in B_{\bar{\rho}}(\bar{y}) \cap[V>\bar{\lambda}]$,

$$
\sup _{\xi \in \partial_{P} V(y)} \liminf _{\lambda \rightarrow 0^{+}}\left\langle\xi, f(y)-A_{\lambda} y\right\rangle+a V(y)+W(y) \leq 0, \quad \sup _{\xi \in \partial_{\infty} V(y)} \liminf _{\lambda \rightarrow 0^{+}}\left\langle\xi, f(y)-A_{\lambda} y\right\rangle \leq 0 .
$$


Indeed, by taking $y_{\lambda}:=J_{\lambda} y$ it follows that $A_{\lambda} y \in A y_{\lambda}, y_{\lambda} \underset{\operatorname{Dom}}{\rightarrow} y$, and, for every $\xi \in \partial_{P} V(y) \cup$ $\partial_{\infty} V(y)$ and $\delta>0$,

$$
\liminf _{\lambda \rightarrow 0^{+}}\left\langle\xi+\delta\left(y-y_{\lambda}\right), f(y)-A_{\lambda} y\right\rangle \leq-\kappa(\xi)(a V(y)+W(y)),
$$

( $\kappa$ was defined in (3.7)) showing that (iv) holds.

(d) A careful reading of the proof of " $(\mathrm{v}) \Longrightarrow(\mathrm{i})$ " in Theorem 3.3 (postponed to Sect. 6) reveals that Theorem 3.3(iv) is also equivalent to, $\forall y \in B_{\bar{\rho}}(\bar{y}) \cap[V>\bar{\lambda}]$ and $\delta>0$,

$$
\begin{aligned}
\sup _{\xi \in \partial_{P} V(y)} & \sup _{\varepsilon>0} \liminf _{z} \inf _{\substack{\operatorname{Dom} A \\
z^{*} \in A_{\varepsilon} z}}\left\langle\xi+\delta(y-z), f(z)-z^{*}\right\rangle+a V(y)+W(y) \leq 0, \\
& \sup _{\xi \in \partial_{\infty} V(y)} \sup _{\varepsilon>0} \liminf _{z \underset{\operatorname{Dom} A}{\longrightarrow} y} \inf _{z^{*} \in A_{\varepsilon} z}\left\langle\xi+y-z, f(z)-z^{*}\right\rangle \leq 0,
\end{aligned}
$$

where $A_{\varepsilon}: H \rightrightarrows H$ is defined by (e.g., Martinez-Legaz \& Théra [26])

$$
y^{*} \in A_{\varepsilon} y \Longleftrightarrow\left\langle y^{*}-z^{*}, y-z\right\rangle \geq-\varepsilon \text { for all }\left(z^{*}, z\right) \in A \text {. }
$$

The following result shows that Theorem 3.3 takes a simple form on $\operatorname{Int}(\operatorname{Dom} A)$. The proof of the statement (iv) $\Longrightarrow$ (i) is postponed to Sect. 6 .

Theorem 3.4. We let $V \in \mathcal{F}_{w}(H), W \in \mathcal{F}^{+}(H)$, and $a \in \mathbb{R}_{+}$be given, and fix $\bar{y} \in \operatorname{Dom} A$, $\bar{\lambda} \in[-\infty, V(\bar{y}))$, and $\bar{\rho} \in(0,+\infty]$ so that

$$
B_{\bar{\rho}}(\bar{y}) \cap[V>\bar{\lambda}] \cap \operatorname{Dom} V \subset \operatorname{Int}(\operatorname{co}(\operatorname{Dom} A)) \neq \emptyset .
$$

Then, the following statements are equivalent:

(i) $\forall x_{0} \in B_{\bar{\rho}}(\bar{y}) \cap[V>\bar{\lambda}] \cap \operatorname{Dom} V$,

$$
e^{a t} V\left(x\left(t ; x_{0}\right)\right)+\int_{0}^{t} W\left(x\left(\tau ; x_{0}\right)\right) d \tau \leq V\left(x_{0}\right) \quad \forall t \in\left[0, \rho\left(x_{0}\right)\right],
$$

where $\rho\left(x_{0}\right)>0$ is defined in (3.6);

(ii) $\forall y \in B_{\bar{\rho}}(\bar{y}) \cap[V>\bar{\lambda}]$ and $\delta>0$,

$$
\sup _{\xi \in \partial_{P} V(y)}\left\langle\xi, f(y)-\Pi_{A y}(f(y))\right\rangle+a V(y)+W(y) \leq 0, \sup _{\xi \in \partial_{\infty} V(y)}\left\langle\xi, f(y)-\Pi_{A y}(f(y))\right\rangle \leq 0
$$

(iii) $\forall y \in B_{\bar{\rho}}(\bar{y}) \cap[V>\bar{\lambda}]$ and $\delta>0$,

$$
\sup _{\xi \in \partial_{P} V(y)} \inf _{y^{*} \in A y}\left\langle\xi, f(y)-y^{*}\right\rangle+a V(y)+W(y) \leq 0, \sup _{\xi \in \partial_{\infty} V(y)} \inf _{y^{*} \in A y}\left\langle\xi, f(y)-y^{*}\right\rangle \leq 0 ;
$$

(iv) $\forall y \in B_{\bar{\rho}}(\bar{y}) \cap[V>\bar{\lambda}]$ and $(\xi, \eta) \in \widetilde{\mathrm{N}}_{\mathrm{epi} V}^{P}(y, V(y))$,

$$
\inf _{y^{*} \in A y}\left\langle\xi, f(y)-y^{*}\right\rangle \leq \eta(a V(y)+W(y)) .
$$

Proof. The proof of (iv) $\Longrightarrow$ (i) is postponed to Sect. 6. Since that the statements (ii) $\Longrightarrow$ (iii) $\Longrightarrow$ (iv) are obvious, we only need to show that (i) $\Longrightarrow$ (ii) holds. To achieve this goal, we may first assume that $W$ is Lipschitz continuous on the bounded subsets of $H$, according to 
Lemma 3.1. Next, we fix $y \in B_{\bar{\rho}}(\bar{y}) \cap[V>\bar{\lambda}],(\xi,-\kappa(\xi)) \in \mathrm{N}_{\text {epi } V}^{P}(y, V(y))$ ( $\kappa$ was defined in (3.7)), and $\delta>0$, so that $y \in \operatorname{Dom} V \cap \operatorname{Dom} A$. Arguing as in the proof of Theorem 3.3, by (i) we let $T>0$ and $\alpha>0$ be such that, for all $t \in[0, T]$,

$$
\langle\xi, x(t ; y)-y\rangle-\alpha\|x(t ; y)-y\|^{2} \leq \kappa(\xi)\left(\left(e^{-a t}-1\right) V(y)-e^{-a t} \int_{0}^{t} W(x(\tau ; y)) d \tau\right)=: h(t) .
$$

Then, because $x(\cdot ; y)$ is a strong solution (remember that $y \in \operatorname{Dom} A$ ), multiplying the inequality above by $t^{-1}$ and next taking the limit as $t \rightarrow 0^{+}$we obtain that

$$
\left\langle\xi, f(y)-\Pi_{A y}(f(y))\right\rangle=\left\langle\xi, \frac{d^{+}}{d t} x(0, y)\right\rangle \leq-\kappa(\xi)(a V(y)+W(y)),
$$

establishing (ii).

Now, using the concept of Dini directional derivative, we give the primal counterpart of Theorem 3.3.

THEOREM 3.5. With the notation of Theorem 3.3 we assume that, for every $y \in B_{\bar{\rho}}(\bar{y}) \cap[V>$ $\bar{\lambda}] \cap \operatorname{Dom} V$ and $\delta>0$,

$$
\liminf _{z \rightarrow \operatorname{Dom}_{A}} \inf _{z^{*} \in A z} V^{\prime}\left(y, f(z)-z^{*}\right)+\delta\left\langle z-y, z^{*}\right\rangle+a V(y)+W(y) \leq 0 .
$$

Then, for every $x_{0} \in \operatorname{Dom} V \cap B_{\bar{\rho}}(\bar{y}) \cap[V>\bar{\lambda}]$,

$$
e^{a t} V\left(x\left(t ; x_{0}\right)\right)+\int_{0}^{t} W\left(x\left(\tau ; x_{0}\right)\right) d \tau \leq V\left(x_{0}\right) \quad \forall t \in\left[0, \rho\left(x_{0}\right)\right]
$$

where $\rho\left(x_{0}\right)>0$ is defined in (3.6).

Proof. It is sufficient to verify Theorem 3.3(iv). Let $y \in B_{\bar{\rho}}(\bar{y}) \cap[V>\bar{\lambda}], \delta>0$, and $\xi \in$ $\partial_{P} V(y) \cup \partial_{\infty} V(y)$ so that $(\xi,-\kappa(\xi)) \in \mathrm{N}_{\text {epi } V}^{P}(y, V(y))$, where $\kappa(\cdot)$ is defined in $(3.7)$; hence, $y \in \operatorname{Dom} V$. If $\varepsilon>0$ is given, by the current assumption there exists a sequence $\left(y_{k}, y_{k}^{*}\right)_{k} \subset A$ such that

$$
V^{\prime}\left(y, f\left(y_{k}\right)-y_{k}^{*}\right)+\delta\left\langle y-y_{k}, f\left(y_{k}\right)-y_{k}^{*}\right\rangle+a V(y)+W(y)<\varepsilon, \text { for } k \in \mathbb{N} .
$$

We may distinguish two cases: if $\alpha_{k}:=\left\langle y-y_{k}, f\left(y_{k}\right)-y_{k}^{*}\right\rangle \geq 0$ for infinitely many $k$, then

$$
\left(\begin{array}{c}
f\left(y_{k}\right)-y_{k}^{*} \\
\varepsilon-(\kappa(\xi)-1) \delta \alpha_{k}-a V(y)-W(y)
\end{array}\right) \in \operatorname{epi}\left(V^{\prime}(y, \cdot)\right) \subset \mathrm{T}_{\text {epi } V}(y, V(y)) .
$$

But $\mathrm{T}_{\text {epi } V}(y, V(y))$ is the dual cone of $\mathrm{N}_{\text {epi } V}^{P}(y, V(y))$ (e.g., [19]), and so we write

$$
\begin{aligned}
\left\langle\xi+\delta\left(y-y_{k}\right), f\left(y_{k}\right)-y_{k}^{*}\right\rangle & =\left\langle(\xi,-\kappa(\xi)),\left(f\left(y_{k}\right)-y_{k}^{*}, \varepsilon-(\kappa(\xi)-1) \delta \alpha_{k}-a V(y)-W(y)\right)\right\rangle \\
& +\kappa(\xi)(\varepsilon-a V(y)-W(y))-\kappa(\xi)(\kappa(\xi)-1) \delta \alpha_{k} \\
& \leq-\kappa(\xi)(a V(y)+W(y))+\kappa(\xi) \varepsilon,
\end{aligned}
$$


which yields Theorem 3.3(iv), as $\varepsilon \rightarrow 0$. Otherwise, if $\alpha_{k} \leq 0$ for all $k$, we obtain that

$$
\begin{aligned}
\left\langle\xi+\delta\left(y-y_{k}\right), f\left(y_{k}\right)-y_{k}^{*}\right\rangle & =\left\langle(\xi,-\kappa(\xi)),\left(f\left(y_{k}\right)-y_{k}^{*}, \varepsilon-\delta \alpha_{k}-a V(y)-W(y)\right)\right\rangle \\
& +\kappa(\xi)(\varepsilon-a V(y)-W(y))+(1-\kappa(\xi)) \delta \alpha_{k} \\
& \leq-\kappa(\xi)(a V(y)+W(y))+\kappa(\xi) \varepsilon,
\end{aligned}
$$

showing that Theorem 3.3(iv) also holds in this case. Thus, the desired conclusion follows.

REMARK 3.2. It is worth observing that the conclusion of Theorem 3.5 also holds if for every $y \in B_{\bar{\rho}}(\bar{y}) \cap[V>\bar{\lambda}] \cap$ Dom $V$ and $\delta>0$ we have that

$$
\liminf _{\substack{\operatorname{Dom}_{A} \\ A^{*}}} \inf _{z^{*} \in A z} V^{\prime}\left(y, f(y)-z^{*}\right)+\delta\left\langle z-y, z^{*}\right\rangle+a V(y)+W(y) \leq 0 .
$$

From a geometrical point of view, Theorem 3.3 can also be viewed as a criterion for the invariance (or viability $([2,3])$ ) of subsets with respect to $(1.1)$.

Definition 2. A nonempty subset $S \subset H$ is said invariant for (1.1) if for all $x_{0} \in S \cap$ $\operatorname{cl}($ Dom $A)$ we have that $x\left(t ; x_{0}\right) \in S$ for all $t \geq 0$.

In other words, $S$ is invariant for (1.1) if and only if the function $\delta_{S \cap c l(\operatorname{Dom} A)}$ is a Lyapunov function for (1.1); hence, Theorems 3.3 and 3.4 apply and yield:

THEOREM 3.6. Let $S \subset H$ be a weakly closed set. Then, the following conditions are equivalent:

(i) $S$ is invariant for (1.1);

(ii) $\forall y \in S \cap \operatorname{cl}(\operatorname{Dom} A)$,

$$
\sup _{\xi \in \mathrm{N}_{S \cap \mathrm{cl}(\operatorname{Dom} A)}^{L}(y)} \liminf _{\substack{\operatorname{Dom} A}}\left\langle\xi+y-z, f(z)-\Pi_{A z}(f(z))\right\rangle \leq 0 ;
$$

(iii) $\forall y \in S \cap \operatorname{cl}(\operatorname{Dom} A)$,

$$
\sup _{\xi \in \mathrm{N}_{S \operatorname{scl}(\operatorname{Dom} A)}(y)} \liminf _{z_{\operatorname{Dom} A} y} \lim _{\lambda \rightarrow 0^{+}}\left\langle\xi+y-z, f(z)-A_{\lambda}(z+\lambda f(z))\right\rangle \leq 0 ;
$$

(iv) $\forall y \in S \cap \operatorname{cl}(\operatorname{Dom} A)$,

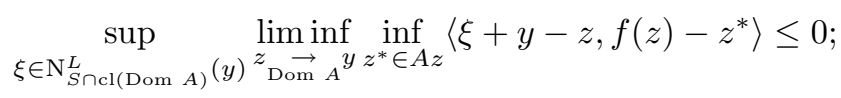

(v) if $S \cap \operatorname{cl}(\operatorname{Dom} A) \subset \operatorname{Int}(\operatorname{co}(\operatorname{Dom} A)), \forall y \in S$,

$$
\sup _{\xi \in \mathrm{N}_{S}^{L}(y)} \inf _{y^{*} \in A y}\left\langle\xi, f(z)-z^{*}\right\rangle \leq 0 .
$$

By the very definition of the weak solution $x\left(\cdot, x_{0}\right)$, it follows that the convex functions $\delta_{\operatorname{cl}(\operatorname{Dom} A)}$ and $d(\cdot, \operatorname{cl}(\operatorname{Dom} A))$ are Lyapunov functions for (1.1). Likewise, the set $\operatorname{cl}(\operatorname{Dom} A)$ is invariant for (1.1). These facts can also be checked by using Theorem 3.3:

Corollary 3.7. Let be given $y \in \operatorname{cl}(\operatorname{Dom} A), \xi \in \mathrm{N}_{\mathrm{cl}(\operatorname{Dom} A)}(y)$, and $\delta>0$. If

$$
y_{\lambda}:=J_{\lambda}(y+\lambda f(y)), y_{\lambda}^{*}:=\lambda^{-1}\left[y+\lambda f(y)-y_{\lambda}\right], \lambda>0,
$$


then $y_{\lambda}^{*} \in A y_{\lambda}, \lim _{\lambda \rightarrow 0^{+}} y_{\lambda} \rightarrow y$, and

$$
\liminf _{\lambda \rightarrow 0^{+}}\left\langle\xi+\delta\left(y-y_{\lambda}\right), f\left(y_{\lambda}\right)-y_{\lambda}^{*}\right\rangle \leq 0 .
$$

Consequently, the conditions (i)-(vi) of Theorem 3.3 hold when $V$ either stands for $\delta_{\mathrm{cl}(\operatorname{Dom} A)}$ or $d(\cdot, \operatorname{cl}(\operatorname{Dom} A)), W=0$, and $a=0$.

Proof. First, we recall that $y_{\lambda}^{*}=A_{\lambda}(y+\lambda f(y)) \in A y_{\lambda}$ for every $\lambda>0$. Hence, invoking the fact that $\frac{1}{\lambda}\left(y-J_{\lambda} y\right) \in A\left(J_{\lambda} y\right)$, by the monotonicity of $A$ we write

$$
\left\langle\lambda^{-1}\left(y-J_{\lambda} y\right)-\lambda^{-1}\left(\lambda f(y)+y-y_{\lambda}\right), J_{\lambda} y-y_{\lambda}\right\rangle \geq 0 .
$$

But $\left\|J_{\lambda} y-y_{\lambda}\right\|^{2} \leq\left\langle f(y), y_{\lambda}-J_{\lambda} y\right\rangle \leq \lambda\|f(y)\|^{2}$ (as $J_{\lambda}$ is nonexpansive), and so $\lim _{\lambda \rightarrow 0^{+}} y_{\lambda}=$ $\lim _{\lambda \rightarrow 0^{+}} J_{\lambda} y=y$. Finally, observing that $\left\langle y-y_{\lambda}, f(y)-y_{\lambda}^{*}\right\rangle=-\lambda^{-1}\left\|y_{\lambda}-y\right\|^{2} \leq 0$, we obtain that

$$
\liminf _{\lambda \rightarrow 0^{+}}\left\langle\xi+\delta\left(y-y_{\lambda}\right), f\left(y_{\lambda}\right)-y_{\lambda}^{*}\right\rangle \leq \liminf _{\lambda \rightarrow 0^{+}}\left\langle\xi, f\left(y_{\lambda}\right)-y_{\lambda}^{*}\right\rangle=\liminf _{\lambda \rightarrow 0^{+}} \lambda^{-1}\left\langle\xi, y_{\lambda}-y\right\rangle \leq 0,
$$

where in the last inequality we used the facts that $\xi \in \mathrm{N}_{\mathrm{cl}(\operatorname{Dom} A)}(y)$ and $y_{\lambda} \in \operatorname{Dom} A$. Hence, the desired conclusion follows since $\partial_{\infty} d(y, \operatorname{cl}(\operatorname{Dom} A))=\{\theta\}\left(\subset \mathrm{N}_{\mathrm{cl}(\operatorname{Dom} A)}(y)\right)$ and

$$
\partial_{P} \delta_{\mathrm{cl}(\operatorname{Dom} A)}(y)=\partial_{\infty} \delta_{\mathrm{cl}(\operatorname{Dom} A)}(y)=\mathrm{N}_{\mathrm{cl}(\operatorname{Dom} A)}(y), \partial_{P} d(y, \operatorname{cl}(\operatorname{Dom} A)) \subset \mathrm{N}_{\mathrm{cl}(\operatorname{Dom} A)}(y),
$$

which completes the proof of the current corollary.

4. Illustrative examples. To illustrate the previous results, we exhibit some concrete examples of Lyapunov pairs. Throughout this section, $\Omega \subset \mathbb{R}^{n}$ is an open bounded set with a sufficiently smooth boundary $\partial \Omega$, and $\left(L^{p}(\Omega),\|\cdot\|_{p}\right)$ is the usual normed space of Lebesgue $p$-integrable functions; for simplicity, if $p=2$, instead of $\|\cdot\|_{2}$ we write $\|\cdot\|$ and denote by $\langle\cdot, \cdot\rangle$ the corresponding inner product. We denote $\left(L^{2}(\Omega)\right)^{n}$ the Hilbert space endowed with the inner product $\left\langle\left(u_{1}, \cdots, u_{n}\right),\left(v_{1}, \cdots, v_{n}\right)\right\rangle:=\left\langle u_{1}, v_{1}\right\rangle+\cdots+\left\langle u_{n}, v_{n}\right\rangle$ and the norm $\left\|\left(u_{1}, \cdots, u_{n}\right)\right\|:=$ $\left(\left\|u_{1}\right\|^{2}+\cdots+\left\|u_{n}\right\|^{2}\right)^{\frac{1}{2}}$, for $\left(u_{1}, \cdots, u_{n}\right),\left(v_{1}, \cdots, v_{n}\right) \in\left(L^{2}(\Omega)\right)^{n}$. The Euclidean norm in $\mathbb{R}^{n}$ is denoted by $|\cdot|$. If $k \geq 0$ is an integer and $1 \leq p \leq \infty, H^{k}(\Omega)$ is the Sobolev space of functions $u$ on the open set $\Omega \subset \mathbb{R}^{n}$ for which $D^{\alpha} u$ belong to $L^{2}(\Omega)$ when $|\alpha| \leq k$, with its usual norm. $H_{0}^{k}(\Omega)$ is the closure of $C_{0}^{\infty}(\Omega)$ in $H^{k}(\Omega)$. We denote by c $(n, \Omega)$ a (positive) Poincaré's constant; that is, a constant depending only on $\Omega$ and the dimension $n$ such that

$$
\mathrm{c}(n, \Omega)\|u\|^{2} \leq\|\nabla u\|^{2} \text { for every } u \in H_{0}^{1}(\Omega) .
$$

We consider a typical simple example of a partial differential equation (PDE, for short) given by

$$
\begin{cases}\dot{u}-\Delta u=\rho(\|u\|) u & \text { in }(0,+\infty) \times \Omega \\ u(t ; x)=0 & \text { in }(0,+\infty) \times \partial \Omega \\ u(0, x)=u_{0}(x) \in L^{2}(\Omega) & \text { in } \Omega,\end{cases}
$$

where $\Delta$ is the Laplacian operator (with respect to the state variable $x$ ), and $\rho: \mathbb{R} \rightarrow \mathbb{R}$ is a given function such that the mapping $f: L^{2}(\Omega) \rightarrow L^{2}(\Omega)$ defined as

$$
f(u):=\rho(\|u\|) u
$$


is Lipschitz continuous. The PDE in (4.2) can be rewritten in the form of (1.1) by defining the operator $A: L^{2}(\Omega) \rightrightarrows L^{2}(\Omega)$ as

$$
A u:=-\Delta u, \operatorname{Dom} A=\left\{u \in H_{0}^{1}(\Omega) \mid \Delta u \in L^{2}(\Omega)\right\}=H_{0}^{1}(\Omega) \cap H^{2}(\Omega) .
$$

It is well established, e.g. Brezis [12] for example, that $A$ is a self-adjoint maximally monotone operator with $\operatorname{cl}(\operatorname{Dom} A)=L^{2}(\Omega)$ (the closure is taken with respect to the $L^{2}(\Omega)$-norm).

Given an integer $p \geq 1$, if we denote $A^{p}: L^{2}(\Omega) \rightrightarrows L^{2}(\Omega)$ the $p$ times composition of $A$ with itself, we know, for instance from Brezis [13, Theorem IX.25], that

$$
\operatorname{Dom} A^{p}=\left\{u \in H^{2 p}(\Omega) \mid u=\Delta u=\cdots=\Delta^{p-1} u=0 \text { on } \partial \Omega\right\} .
$$

We set $A^{0}$ for the identity mapping in $L^{2}(\Omega)$, and we define for $p \geq 0$ the functions $V_{p}, W_{p}$ : $L^{2}(\Omega) \rightarrow \mathbb{R}_{+} \cup\{+\infty\}$ as

$$
\begin{gathered}
V_{p}(u):= \begin{cases}\frac{1}{2}\left\|A^{p} u\right\|^{2} & \text { if } u \in \text { Dom } A^{p} \\
+\infty & \text { otherwise, }\end{cases} \\
W_{p}(u):= \begin{cases}\frac{1}{2}\left\|\nabla\left(A^{p} u\right)\right\|^{2} & \text { if } u \in H^{2 p+1}(\Omega) \text { and } \Delta^{q} u_{\mid \partial \Omega}=0 \text { for all } q=0,1, \cdots, p \\
+\infty & \text { otherwise. }\end{cases}
\end{gathered}
$$

Then, clearly Dom $V_{p}=\operatorname{Dom} A^{p}$ and $\operatorname{Dom} W_{p}=\left\{u \in H^{2 p+1}(\Omega)\right) \mid \Delta^{q} u_{\mid \partial \Omega}=0$ for all $q=$ $0,1, \cdots, p\}$ are convex subsets of $\operatorname{cl}(\operatorname{Dom} A)$, and it can also be checked that both functions $V_{p}, W_{p}$ are proper, convex, and (weakly) lsc on $L^{2}(\Omega)$, at the same time as

$$
\begin{aligned}
V_{p}^{\prime}(u ; v) & =\left\langle\Delta^{p} u, \Delta^{p} v\right\rangle \text { for all } u, v \in \text { Dom } V_{p}, \\
W_{p}^{\prime}(u ; v) & =\left\langle\nabla\left(\Delta^{p} u\right), \nabla\left(\Delta^{p} v\right)\right\rangle \text { for all } u, v \in \operatorname{Dom} W_{p} .
\end{aligned}
$$

In particular, we have that $A=\partial W_{0}$ (see, e.g., [4]) and, so, for every $u_{0} \in L^{2}(\Omega)$ and $T>0$ equation (4.2) has a unique strong solution $u\left(\cdot ; u_{0}\right) \in \mathcal{C}\left([0, T] ; L^{2}(\Omega)\right)$, which satisfies $u\left(t ; u_{0}\right) \in$ Dom $A$ for every $t \in(0, T]$.

In the two examples below, we investigate concrete Lyapunov pairs with respect to (4.2) by assuming some boundedness conditions on the function $\rho$. These examples have been studied in the case $\rho=0$ in $[30]$ to provide some regularity properties of the solution of a general variant of (4.2). Our main objective in the next examples is to show how our criteria can be applied, namely even if in this situation it holds that Dom $V_{p} \subset$ Dom $A$ for every $p \geq 1$ and Dom $W_{p} \subset$ Dom $A$ for every $p \geq 0$, then it seems necessary to use approximate sequences to check the criteria of Theorem 3.3.

EXAmPle 4.1. Given $p \in \mathbb{N}$, we suppose that the function $\rho$ satisfies

$$
\sup \rho \leq \frac{\mathrm{c}(n, \Omega)}{4}
$$

Then, we claim that the pairs $\left(V_{p}, \frac{1}{2} W_{p}\right)$ and $\left(W_{p}, \frac{1}{2} V_{p+1}\right)$ form Lyapunov pairs with respect to (4.2).

Proof. We fix $p \geq 0, u \in \operatorname{Dom} V_{p}$, and $\delta>0$, and for $\lambda>0$ we denote

$$
u_{\lambda}:=u+\lambda \Delta u_{\lambda}=(I-\lambda \Delta)^{-1}(u),
$$


so that $f\left(u_{\lambda}\right) \in \operatorname{Dom} V_{p}, \Delta^{p} u_{\lambda} \in H_{0}^{1}(\Omega)$, and $\Delta^{p+1} u_{\lambda} \in L^{2}(\Omega)$. Moreover, since $u_{\lambda} \rightarrow u$ as $\lambda \rightarrow 0^{+}$, by the current assumption on the function $\rho$ we may suppose that

$$
\lambda \rho\left(\left\|u_{\lambda}\right\|\right) \leq \frac{1}{2} .
$$

Now, using (4.5) and (4.7), we write

$$
\begin{aligned}
& V_{p}^{\prime}\left(u ; f\left(u_{\lambda}\right)+\Delta u_{\lambda}\right)=\rho\left(\left\|u_{\lambda}\right\|\right)\left\langle\Delta^{p} u_{\lambda}-\lambda \Delta^{p+1} u_{\lambda}, \Delta^{p} u_{\lambda}\right\rangle+\left\langle\Delta^{p} u_{\lambda}, \Delta^{p+1} u_{\lambda}\right\rangle-\lambda\left\|\Delta^{p+1} u_{\lambda}\right\|^{2} \\
& =\rho\left(\left\|u_{\lambda}\right\|\right)\left\|\Delta^{p} u_{\lambda}\right\|^{2}+\left(\frac{1}{2}-\lambda \rho\left(\left\|u_{\lambda}\right\|\right)\right)\left\langle\Delta^{p+1} u_{\lambda}, \Delta^{p} u_{\lambda}\right\rangle+\frac{1}{2}\left\langle\Delta^{p} u_{\lambda}, \Delta^{p+1} u_{\lambda}\right\rangle-\lambda\left\|\Delta^{p+1} u_{\lambda}\right\|^{2} .
\end{aligned}
$$

By the monotonicity of $A=-\Delta$ and the obvious fact $A 0=0$ we have that

$$
\left\langle\Delta^{p+1} u_{\lambda}, \Delta^{p} u_{\lambda}\right\rangle=-\left\langle A\left(\Delta^{p} u_{\lambda}\right), \Delta^{p} u_{\lambda}\right\rangle \leq 0 .
$$

Hence, in view of (4.8) we derive that

$$
\left(\frac{1}{2}-\lambda \rho\left(\left\|u_{\lambda}\right\|\right)\right)\left\langle\Delta^{p+1} u_{\lambda}, \Delta^{p} u_{\lambda}\right\rangle \leq 0
$$

and therefore, using the last estimate,

$$
V_{p}^{\prime}\left(u ; f\left(u_{\lambda}\right)+\Delta u_{\lambda}\right) \leq \rho\left(\left\|u_{\lambda}\right\|\right)\left\|\Delta^{p} u_{\lambda}\right\|^{2}+\frac{1}{2}\left\langle\Delta^{p} u_{\lambda}, \Delta^{p+1} u_{\lambda}\right\rangle .
$$

Now, by the Green formula we know that $\left\langle\Delta^{p} u_{\lambda}, \Delta^{p+1} u_{\lambda}\right\rangle=-\left\langle\nabla\left(\Delta^{p} u_{\lambda}\right), \nabla\left(\Delta^{p} u_{\lambda}\right)\right\rangle$. As a result, the observation (4.9) and the current assumption $\sup \rho \leq \frac{\mathrm{c}(n, \Omega)}{4}$, together with (4.1) (recall that $\left.\Delta^{p} u_{\lambda} \in H_{0}^{1}(\Omega)\right)$ and the fact $\delta\left\langle u-u_{\lambda}, \Delta u_{\lambda}\right\rangle=-\delta \lambda^{-1}\left\|u-u_{\lambda}\right\|^{2} \leq 0$, yield to

$$
\begin{aligned}
V_{p}^{\prime}\left(u ; f\left(u_{\lambda}\right)+\Delta u_{\lambda}\right)+\delta\left\langle u-u_{\lambda}, \Delta u_{\lambda}\right\rangle & \leq \rho\left(\left\|u_{\lambda}\right\|\right)\left\|\Delta^{p} u_{\lambda}\right\|^{2}-\frac{1}{4}\left\|\nabla\left(\Delta^{p} u_{\lambda}\right)\right\|^{2}-\frac{1}{4}\left\|\nabla\left(\Delta^{p} u_{\lambda}\right)\right\|^{2} \\
& \leq\left(\rho\left(\left\|u_{\lambda}\right\|\right)-\frac{\mathrm{c}(n, \Omega)}{4}\right)\left\|\Delta^{p} u_{\lambda}\right\|^{2}-\frac{1}{4}\left\|\nabla\left(\Delta^{p} u_{\lambda}\right)\right\|^{2} \\
& \leq-\frac{1}{4}\left\|\nabla\left(\Delta^{p} u_{\lambda}\right)\right\|^{2}=-\frac{1}{2} W_{p}\left(u_{\lambda}\right) ;
\end{aligned}
$$

the last equality holds because $u_{\lambda} \in$ Dom $W_{p}$. Invoking the lower semicontinuity of $W_{p}$, Theorem 3.5 applies and entails that $\left(V_{p}, \frac{1}{2} W_{p}\right)$ forms a Lyapunov pairs for (4.2).

To prove that $\left(W_{p}, \frac{1}{2} V_{p+1}\right)$ is a Lyapunov pair, we take $u \in \operatorname{Dom} W_{p}$ and let $\left(u_{\lambda}\right)_{\lambda>0}$ be defined as in (4.7). Then, arguing as above, we observe that $u_{\lambda}, f\left(u_{\lambda}\right), \Delta u_{\lambda} \in \operatorname{Dom} W_{p}$ so that (4.6) reads

$$
\begin{aligned}
W_{p}^{\prime}\left(u ; f\left(u_{\lambda}\right)+\Delta u_{\lambda}\right) & =\left\langle\nabla\left(\Delta^{p} u\right), \nabla\left(\Delta^{p}\left(f\left(u_{\lambda}\right)\right)\right)\right\rangle+\left\langle\nabla\left(\Delta^{p} u\right), \nabla\left(\Delta^{p+1} u_{\lambda}\right)\right\rangle \\
& =\rho\left(\left\|u_{\lambda}\right\|\right)\left\|\nabla\left(\Delta^{p} u_{\lambda}\right)\right\|^{2}+\left(\lambda \rho\left(\left\|u_{\lambda}\right\|\right)-\frac{1}{2}\right)\left\|\Delta^{p+1} u_{\lambda}\right\|^{2}-\frac{1}{2}\left\|\Delta^{p+1} u_{\lambda}\right\|^{2} .
\end{aligned}
$$


Thus, by (4.1), (4.8), and the current assumption on the function $\rho$,

$$
\begin{aligned}
W_{p}^{\prime}\left(u ; f\left(u_{\lambda}\right)+\Delta u_{\lambda}\right)+\delta\left\langle u-u_{\lambda}, \Delta u_{\lambda}\right\rangle & \leq\left(\rho\left(\left\|u_{\lambda}\right\|\right)-\frac{\mathrm{c}(n, \Omega)}{4}\right)\left\|\nabla\left(\Delta^{p} u_{\lambda}\right)\right\|^{2}-\frac{1}{4}\left\|\Delta^{p+1} u_{\lambda}\right\|^{2} \\
& \leq-\frac{1}{4}\left\|\Delta^{p+1} u_{\lambda}\right\|^{2}=-\frac{1}{2} V_{p+1}\left(u_{\lambda}\right) .
\end{aligned}
$$

Hence, by Theorem 3.5 we deduce that $\left(W_{p}, \frac{1}{2} V_{p+1}\right)$ forms a Lyapunov pair for (4.2).

Let us introduce the (lsc convex proper) functions $V_{p, q}: L^{2}(\Omega) \rightarrow \mathbb{R}_{+} \cup\{+\infty\}$, where $p \in[2,+\infty)$ and $q \in \mathbb{N}^{*}$, defined as

$$
V_{p, q}(u):= \begin{cases}\frac{1}{p}\left\|\Delta^{q} u\right\|_{L^{p}(\Omega)}^{p} & \text { if } u \in \operatorname{Dom} A^{q} \text { and } \Delta^{q} u \in L^{p}(\Omega), \\ +\infty & \text { otherwise }\end{cases}
$$

where the operator $A^{q}$ is defined in (4.4). It can be checked that for every $u, v \in \operatorname{dom} V_{p, q}=$ $\left\{u \in \operatorname{Dom} A^{q} \mid \Delta^{q} u \in L^{p}(\Omega)\right\}$ one has that

$$
V_{p, q}^{\prime}(u ; v)=\left\langle\left|\Delta^{q} u\right|^{p-1} \operatorname{sign}\left(\Delta^{q} u\right), \Delta^{q} v\right\rangle .
$$

EXAmPLE 4.2. Given $p \in[2,+\infty)$ and $q \in \mathbb{N}^{*}$, we suppose that

$$
\sup \rho \leq 2 \mathrm{c}(n, \Omega)(p-1) p^{-2} .
$$

Then, we claim that:

(i) for $a_{1}:=\frac{2 \mathrm{c}(n, \Omega)(p-1)}{p}$, the pair $\left(V_{p, q}, a_{1} V_{p, q}\right)$ forms a Lyapunov pair for (4.2);

(ii) if $n \geq 3$, there exists an $a_{2}>0$ depending only on $n$ and $\Omega$ such that the pair of functions $\left(V_{p, q}, a_{2}\left(V_{p n(n-2)^{-1}, q}\right)^{\frac{n-2}{n}}\right)$ forms a Lyapunov pair for (4.2).

Proof. (i) We fix $u \in \operatorname{dom} V_{p, q}$ and let $\left(u_{\lambda}\right)_{\lambda>0}$ be as defined in (4.7). Thus, by the regularity of Dirichlet's problem in $L^{p}$ spaces (e.g. [13, Theorem IX.32]), for each $\lambda>0$ we have that $u_{\lambda}$, $f\left(u_{\lambda}\right), \Delta u_{\lambda} \in \operatorname{dom} V_{p, q}$ and, so,

$$
\begin{aligned}
& V_{p, q}^{\prime}\left(u ; f(u)+\Delta u_{\lambda}\right) \\
& =\rho(\|u\|)\left\|\Delta^{q} u\right\|_{p}^{p}+\lambda^{-1}\left\langle\left|\Delta^{q} u\right|^{p-1} \operatorname{sign}\left(\Delta^{q} u\right)-\left|\Delta^{q} u_{\lambda}\right|^{p-1} \operatorname{sign}\left(\Delta^{q} u_{\lambda}\right), \Delta^{q} u_{\lambda}-\Delta^{q} u\right\rangle \\
& +\left\langle\left|\Delta^{q} u_{\lambda}\right|^{p-1} \operatorname{sign}\left(\Delta^{q} u_{\lambda}\right), \Delta^{q+1} u_{\lambda}\right\rangle .
\end{aligned}
$$

Since the function $\alpha \mapsto|\alpha|^{p-1} \operatorname{sign}(\alpha)$ is nondecreasing it follows that

$$
\left\langle\left|\Delta^{q} u\right|^{p-1} \operatorname{sign}\left(\Delta^{q} u\right)-\left|\Delta^{q} u_{\lambda}\right|^{p-1} \operatorname{sign}\left(\Delta^{q} u_{\lambda}\right), \Delta^{q} u_{\lambda}-\Delta^{q} u\right\rangle \leq 0,
$$

and so, by the last inequality,

$$
V_{p, q}^{\prime}\left(u ; f(u)+\Delta u_{\lambda}\right) \leq \rho(\|u\|)\left\|\Delta^{q} u\right\|_{p}^{p}+\left\langle\left|\Delta^{q} u_{\lambda}\right|^{p-1} \operatorname{sign}\left(\Delta^{q} u_{\lambda}\right), \Delta^{q+1} u_{\lambda}\right\rangle .
$$

In virtue of the Green formula, we have that

$$
\left\langle\left|\Delta^{q} u_{\lambda}\right|^{p-1} \operatorname{sign}\left(\Delta^{q} u_{\lambda}\right), \Delta^{q+1} u_{\lambda}\right\rangle=-\left\langle\nabla\left(\left|\Delta^{q} u_{\lambda}\right|^{p-1} \operatorname{sign}\left(\Delta^{q} u_{\lambda}\right)\right), \nabla\left(\Delta^{q} u_{\lambda}\right)\right\rangle,
$$


and so

$$
\begin{aligned}
V_{p, q}^{\prime}\left(u ; f(u)+\Delta u_{\lambda}\right) & \leq \rho(\|u\|)\left\|\Delta^{q} u\right\|_{p}^{p}-(p-1)\left\langle\left|\Delta^{q} u_{\lambda}\right|^{p-2} \nabla\left(\Delta^{q} u_{\lambda}\right), \nabla\left(\Delta^{q} u_{\lambda}\right)\right\rangle \\
& =\rho(\|u\|)\left\|\Delta^{q} u\right\|_{p}^{p}-(p-1) \sum_{i=1, \cdots, n} \int_{\Omega}\left|\left(\Delta^{q} u_{\lambda}\right)^{\frac{p}{2}-1} \partial_{i}\left(\Delta^{q} u_{\lambda}\right)\right|^{2} \\
& =\rho(\|u\|)\left\|\Delta^{q} u\right\|_{p}^{p}-4(p-1) p^{-2} \int_{\Omega}\left|\nabla\left(\left(\Delta^{q} u_{\lambda}\right)^{\frac{p}{2}}\right)\right|_{2}^{2} \\
& =\rho(\|u\|)\left\|\Delta^{q} u\right\|_{p}^{p}-4(p-1) p^{-2}\left\|\nabla\left(\left(\Delta^{q} u_{\lambda}\right)^{\frac{p}{2}}\right)\right\|^{2} .
\end{aligned}
$$

Thus, applying (4.1) to $\left(\Delta^{q} u_{\lambda}\right)^{\frac{p}{2}}$ (we remember that $u_{\lambda} \in \operatorname{dom} V_{p, q}$ so that $\Delta^{q} u_{\lambda} \in L^{p}(\Omega)$ and $\left(\Delta^{q} u_{\lambda}\right)^{\frac{p}{2}} \in L^{2}(\Omega)$; while the relation $\nabla\left(\left(\Delta^{q} u_{\lambda}\right)^{\frac{p}{2}}\right) \in L^{2}(\Omega)$ is a consequence of the last equality above) we write

$$
\mathrm{c}(n, \Omega)\left\|\left(\Delta^{q} u_{\lambda}\right)^{\frac{p}{2}}\right\|^{2} \leq\left\|\nabla\left(\left(\Delta^{q} u_{\lambda}\right)^{\frac{p}{2}}\right)\right\|^{2}
$$

and so (4.10) infers

$$
V_{p, q}^{\prime}\left(u ; f(u)+\Delta u_{\lambda}\right) \leq \rho(\|u\|)\left\|\Delta^{q} u\right\|_{p}^{p}-4 \mathrm{c}(n, \Omega)(p-1) p^{-2}\left\|\Delta^{q} u_{\lambda}\right\|_{p}^{p} .
$$

By the lower semicontinuity of $V_{p, q}$ we have that $\left\|\Delta^{q} u\right\|_{p}^{p}=p V(u) \leq \liminf _{\lambda \rightarrow 0^{+}} p V\left(u_{\lambda}\right)=$ $\left\|\Delta^{q} u_{\lambda}\right\|_{p}^{p}$. Thus, taking limits in the last inequality, and making use of the current assumption on the function $\rho$,

$$
\begin{aligned}
\liminf _{\lambda \rightarrow 0^{+}} V_{p, q}^{\prime}\left(u ; f(u)+\Delta u_{\lambda}\right)+2 \mathrm{c}(n, \Omega)(p-1) p^{-2}\left\|\Delta^{q} u\right\|_{p}^{p} & \\
& \leq\left(\rho(\|u\|)-2 \mathrm{c}(n, \Omega)(p-1) p^{-2}\right)\left\|\Delta^{q} u\right\|_{p}^{p} \leq 0 .
\end{aligned}
$$

Consequently, as $\delta\left\langle u-u_{\lambda}, \Delta u_{\lambda}\right\rangle=-\delta\left\|u-u_{\lambda}\right\|^{2} \leq 0$, we obtain

$$
\liminf _{\operatorname{dom} A \ni v \rightarrow u} V_{p, q}^{\prime}(u ; f(u)+\Delta v)+\delta\langle u-v, \Delta v\rangle+2 \mathrm{c}(n, \Omega)(p-1) p^{-1} V_{p, q}(v) \leq 0,
$$

which in view of Theorem 3.5 (together with Remark 3.2) achieves the proof of the statement (i).

(ii) In the case where $n \geq 3$, applying the Sobolev inequality to $\left(\Delta^{q} u_{\lambda}\right)^{\frac{p}{2}}$ we also find a constant $c_{2}>0$, also depending only on $n$ and $\Omega$, such that

$$
c_{2}\left(\int_{\Omega}\left|\left(\Delta^{q} u_{\lambda}\right)^{\frac{p}{2}}\right|^{2 n(n-2)^{-1}}\right)^{(n-2)(2 n)^{-1}} \leq\left(\int_{\Omega}\left|\nabla\left(\left(\Delta^{q} u_{\lambda}\right)^{\frac{p}{2}}\right)\right|^{2}\right)^{\frac{1}{2}} .
$$


Hence, using once again (4.11), from (4.10) gives that

$$
\begin{aligned}
& V_{p, q}^{\prime}\left(u ; f(u)+\Delta u_{\lambda}\right) \\
& \leq \rho(\|u\|)\left\|\Delta^{q} u\right\|_{p}^{p}-2(p-1) p^{-2}\left\|\nabla\left(\left(\Delta^{q} u_{\lambda}\right)^{\frac{p}{2}}\right)\right\|^{2}-2(p-1) p^{-2}\left\|\nabla\left(\left(\Delta^{q} u_{\lambda}\right)^{\frac{p}{2}}\right)\right\|^{2} \\
& \leq \rho(\|u\|)\left\|\Delta^{q} u\right\|_{p}^{p}-2 \mathrm{c}(n, \Omega)(p-1) p^{-2}\left\|\Delta^{q} u_{\lambda}\right\|_{p}^{p}-2 c_{2}(p-1) p^{-2}\left(\int_{\Omega}\left|\left(\Delta^{q} u_{\lambda}\right)^{\frac{p}{2}}\right|^{\frac{2 n}{n-2}}\right)^{\frac{n-2}{n}} \\
& =\rho(\|u\|)\left\|\Delta^{q} u\right\|_{p}^{p}-2 \mathrm{c}(n, \Omega)(p-1) p^{-2}\left\|\Delta^{q} u_{\lambda}\right\|_{p}^{p} \\
& -2 c_{2}(p-1) p^{-2}\left(\frac{p n}{n-2}\right)^{\frac{n-2}{n}}\left(V_{p n(n-2)^{-1}, q}\left(u_{\lambda}\right)\right)^{\frac{n-2}{n}} .
\end{aligned}
$$

Consequently, taking into account the lsc of $V_{p, q}$ and $V_{p n(n-2)^{-1}, q}\left(\right.$ as $\left.p, p n(n-2)^{-1} \geq 2\right)$, together with the current assumption on the function $\rho$,

$$
\liminf _{\lambda \rightarrow 0^{+}} V_{p, q}^{\prime}\left(u ; f(u)+\Delta u_{\lambda}\right)+2 c_{2}(p-1) p^{-2}\left(\frac{p n}{n-2}\right)^{\frac{n-2}{n}}\left(V_{p n(n-2)^{-1}, q}\left(u_{\lambda}\right)\right)^{\frac{n-2}{n}} \leq 0
$$

so that, applying Theorem 3.5 together with Remark 3.2, statement (ii) follows by taking $a_{2}=$ $2 c_{2}(p-1) p^{-2}\left(\frac{p n}{n-2}\right)^{\frac{n-2}{n}}$.

In the following example we investigate the invariance with respect to (4.2) of the convex subsets $C_{p} \subset L^{2}(\Omega), p \in \mathbb{N}$, given by

$$
C_{0}:=\left\{u \in L^{2}(\Omega) \mid u(x) \geq 0 \text { for } x \in \Omega \text { a.e. }\right\},
$$

and for $p \geq 1$ by

$$
C_{p}:=\left\{\begin{array}{l|l}
u \in L^{2}(\Omega) & \begin{array}{l}
\Delta^{p} u \in L^{2}(\Omega), \Delta^{k} u \in H_{0}^{1}(\Omega) \text { for all } k=0, \cdots, p-1, \\
\int_{\Omega} v \Delta^{p} u \geq 0 \text { for all } v \in L^{2}(\Omega) \text { s.t. } v \geq 0 \text { on } \Omega \text { a.e. }
\end{array}
\end{array}\right\} .
$$

EXAMPLE 4.3. For every integer $p \geq 0$, the set $C_{p}$ is invariant for (4.2).

Proof. We fix $p \in \mathbb{N}, u \in C_{p}$, and $\xi \in \mathrm{N}_{C_{p}}(u)$; hence, as $C_{p}$ is a cone and $\theta \in C_{p}$ we get that $\langle\xi, u\rangle=0$. Next, we consider the sequence $\left(u_{\lambda}\right)_{\lambda>0}$ defined in $(4.7)$; i.e. $u_{\lambda}=(I-\lambda \Delta)^{-1}(u)$, so that $\Delta^{k} u_{\lambda} \in H_{0}^{1}(\Omega)$ for all $k=0, \ldots, p$, and

$$
\int_{\Omega}\left(\Delta^{p} u_{\lambda}\right)^{-} \Delta^{p} u=-\lambda \int_{\Omega}\left(\Delta^{p} u_{\lambda}\right)^{-} \Delta^{p+1} u_{\lambda}-\int_{\Omega}\left|\left(\Delta^{p} u_{\lambda}\right)^{-}\right|^{2},
$$

where $\left(\Delta^{p} u_{\lambda}\right)^{-}:=-\min \left\{\Delta^{p} u_{\lambda}(x), 0\right\}$ is the negative part of $\Delta^{p} u_{\lambda}$. On another hand, according to Tartar [35, Chapt. 4 Lemma 1.1], $\left(\Delta^{p} u_{\lambda}\right)^{-} \in H_{0}^{1}(\Omega)$ and we have that

$$
\frac{\partial}{\partial x_{i}}\left(\Delta^{p} u_{\lambda}\right)^{-}=0 \text { if } \Delta^{p} u_{\lambda} \geq 0, \text { and } \frac{\partial}{\partial x_{i}}\left(\Delta^{p} u_{\lambda}\right)^{-}=-\frac{\partial}{\partial x_{i}}\left(\Delta^{p} u_{\lambda}\right) \text { if } \Delta^{p} u_{\lambda}<0 .
$$


Hence, according to the Green formula we obtain

$$
\begin{aligned}
\int_{\Omega}\left(\Delta^{p} u_{\lambda}\right)^{-} \Delta^{p} u & =-\lambda \int_{\Omega}\left(\Delta^{p} u_{\lambda}\right)^{-} \Delta^{p+1} u_{\lambda}+\int_{\Omega}\left(\Delta^{p} u_{\lambda}\right)^{-} \Delta^{p} u_{\lambda} \\
& =\lambda \int_{\Omega} \nabla\left(\left(\Delta^{p} u_{\lambda}\right)^{-}\right) \nabla\left(\Delta^{p} u_{\lambda}\right)-\int_{\Omega}\left|\left(\Delta^{p} u_{\lambda}\right)^{-}\right|^{2} .
\end{aligned}
$$

Thus, using the decomposition $\Delta^{p} u_{\lambda}=\left(\Delta^{p} u_{\lambda}\right)^{+}-\left(\Delta^{p} u_{\lambda}\right)^{-}$, where $\left(\Delta^{p} u_{\lambda}\right)^{+}$denotes the positive part, and the fact that $\int_{\Omega} \nabla\left(\left(\Delta^{p} u_{\lambda}\right)^{-}\right) \nabla\left(\left(\Delta^{p} u_{\lambda}\right)^{+}\right)=0$ (remember $\left.(4.12)\right)$,

$$
\begin{aligned}
\int_{\Omega}\left(\Delta^{p} u_{\lambda}\right)^{-} \Delta^{p} u & =\lambda \int_{\Omega} \nabla\left(\left(\Delta^{p} u_{\lambda}\right)^{-}\right) \nabla\left(\left(\Delta^{p} u_{\lambda}\right)^{+}\right)-\lambda \int_{\Omega}\left|\nabla\left(\left(\Delta^{p} u_{\lambda}\right)^{-}\right)\right|^{2}-\int_{\Omega}\left|\left(\Delta^{p} u_{\lambda}\right)^{-}\right|^{2} \\
& =-\lambda \int_{\Omega}\left|\nabla\left(\left(\Delta^{p} u_{\lambda}\right)^{-}\right)\right|^{2}-\int_{\Omega}\left|\left(\Delta^{p} u_{\lambda}\right)^{-}\right|^{2} .
\end{aligned}
$$

Consequentely, as $\int_{\Omega}\left(\Delta^{p} u_{\lambda}\right)^{-} \Delta^{p} u \geq 0$ (remember that $u \in C_{p}$ ) it infers that $\left(\Delta^{p} u_{\lambda}\right)^{-}(x)=0$, for $x \in \Omega$ a.e., and so $u_{\lambda} \in C_{p}$. Finally, observing that $\langle\xi, f(u)\rangle=\rho(\|u\|)\langle\xi, u\rangle=0$, by the definition of $\xi$ it follows that

$$
\left\langle\xi, f(u)+\Delta u_{\lambda}\right\rangle \leq \lambda^{-1}\left\langle\xi, u_{\lambda}-u\right\rangle \leq 0
$$

Thus, the desired conclusion follows from Theorem 3.6.

ExAmple 4.4. (Continuation of Example 3.1) For fixed $p \in \mathbb{N}$, we consider the dynamical systems given in $\mathbb{R}$ by

$$
\dot{x}_{p}\left(t ; x_{0}\right) \in-\operatorname{Sign}\left(x_{p}\left(t ; x_{0}\right)\right)+p\left|x_{p}\left(t ; x_{0}\right)\right| \text { a.e. } t \geq 0, x_{p}\left(0 ; x_{0}\right)=x_{0} \in \mathbb{R} .
$$

We define the functions $V_{p}: \mathbb{R} \rightarrow \mathbb{R} \cup\{+\infty\}$ as $V_{0}(x):=|x|$ and, if $p>0$,

$$
V_{p}(x):=|x| \text { if }|x| \leq \frac{1}{p} \text { and } V_{p}(x)=+\infty \text { otherwise. }
$$

Then, $V_{p}$ is a Lyapunov function with respect to the system above.

Proof. We fix $x \in \mathbb{R}$. Because each $V_{p}$ is convex $\partial_{P} V_{p}(x)=\partial V_{p}(x)$ and, so, $\partial V_{0}(x)=\{\operatorname{sign}(x)\}$, $\partial_{\infty} V_{0}(x)=\{0\}$

$$
\partial V_{p}(x)=\left\{\begin{array}{ll}
\{\operatorname{sign}(x)\} & \text { if }|x|<\frac{1}{p} \\
{[1,+\infty)} & \text { if } x=\frac{1}{p} \\
(-\infty,-1] & \text { if } x=-\frac{1}{p} \\
\emptyset & \text { if } x \notin\left[-\frac{1}{p}, \frac{1}{p}\right] .
\end{array} \quad, \quad \partial_{\infty} V_{p}(x)= \begin{cases}\{0\} & \text { if }|x|<\frac{1}{p} \\
{[0,+\infty)} & \text { if } x=\frac{1}{p} \\
(-\infty, 0] & \text { if } x=-\frac{1}{p} \\
\emptyset & \text { if } x \notin\left[-\frac{1}{p}, \frac{1}{p}\right] .\end{cases}\right.
$$

Then, since that

$$
\begin{aligned}
\left\langle\partial V_{0}(x),-\operatorname{Sign}(x)\right\rangle & =\langle\operatorname{Sign}(x),-\operatorname{Sign}(x)\rangle=-(\operatorname{Sign}(x))^{2} \leq 0, \\
\left\langle\partial V_{p}(x),-\operatorname{Sign}(x)+p|x|\right\rangle & =(\operatorname{Sign}(x))^{2}(p x-1) \leq 0 \text { if } p>0 \text { and } p x<1, \\
\langle\alpha,-\operatorname{Sign}(x)+p|x|\rangle & =0 \text { for } \alpha \in[0,+\infty) \text { and } x=\frac{1}{p}, \text { and } \\
\langle\alpha,-\operatorname{Sign}(x)+p|x|\rangle & =2 \alpha \leq 0 \text { for } \alpha \in(-\infty, 0] \text { and } x=-\frac{1}{p},
\end{aligned}
$$


Theorem 3.3(iv) applies and yields the desired conclusion.

EXAMPLE 4.5. We consider the differential inclusion given in $\mathbb{R}$ by

$$
\dot{x} \in d(x,[0,1 / 4])(x-1)-\mathrm{N}_{\mathbb{R}_{+}}(x),
$$

where $d(\cdot,[0,1 / 4])$ is the usual distance to $[0,1 / 4]$. Then, the set $[0,1 / 4]$ is stable in the following sense: for every $\varepsilon>0$, there exists $\delta>0$ such that, for every $\left.\left.x_{0} \in\right] 1 / 4,1 / 2\right]$,

$$
x_{0}-1 / 4 \leq \delta \Longrightarrow d\left(x\left(t ; x_{0}\right),[0,1 / 4]\right) \leq \varepsilon \quad \forall t \geq 0 .
$$

Proof. We consider the (convex) lsc proper function $V: \mathbb{R} \rightarrow \mathbb{R} \cup\{+\infty\}$ given by

$$
V(x):=\frac{1}{2} d^{2}(x,[0,1 / 4]) \text { if } x \in[0,1 / 2] \text { and } V(x):=+\infty \text { otherwise, }
$$

so that, for $x \in[0,1 / 2]$,

$$
\partial V(x)=\left\{\begin{array}{l}
0 \text { if } x \in] 0,1 / 4] \\
\left.x-\frac{1}{4} \text { if } x \in\right] 1 / 4,1 / 2\left[, \quad \partial_{\infty} V(x)=\left\{\begin{array}{l}
0 \text { if } x \in] 0,1 / 2[ \\
]-\infty, 0], \text { if } x=0, \\
] 1 / 4,+\infty[, \text { if } x=1 / 2
\end{array} \quad \text { [0, if } x=0\right.\right.
\end{array}\right.
$$

Then, if $x \in[0,1 / 2], \xi_{1} \in \partial V(x)$ and $\xi_{2} \in \partial_{\infty} V(x)$,

$$
\begin{gathered}
0 \geq\left\langle\xi_{1}, d(x,[0,1 / 4])(x-1)\right\rangle= \begin{cases}0 \in \mathbb{R}_{-} \\
(x-1 / 4)^{2}(x-1) \in \mathbb{R}_{-} & \text {if } x \in[0,1 / 4], \\
\alpha \in \mathbb{R}_{-} & \text {if } x=1 / 2 ; 1 / 4,1 / 2[\end{cases} \\
0 \geq\left\langle\xi_{2}, d(x,[0,1 / 4])(x-1)\right\rangle= \begin{cases}0 \in \mathbb{R}_{-} & \text {if } x \in[0,1 / 2[, \\
\alpha \in \mathbb{R}_{-} & \text {if } x=1 / 2 .\end{cases}
\end{gathered}
$$

In other words, Theorem 3.3(iv) applies and entails that $V$ is a Lyapunov function. Consequently, if $\left.\left.x_{0} \in\right] 1 / 4,1 / 2\right]$, then by Lyapunov's Inequality we obtain that

$$
d\left(x\left(t ; x_{0}\right),[0,1 / 4]\right) \leq x_{0}-1 / 4 \text { for all } t \geq 0 .
$$

Thus, for every given $\varepsilon>0$ the inequality in (4.13) holds with $\delta=\sqrt{2 \varepsilon}$ for $t>0$.

5. Other criteria for Lyapunov pairs. In this section we make a unified review of many other useful criteria for $a$-Lyapunov pairs with respect to (1.1).

The first result extends in our setting [30, Theorem 3.6].

Corollary 5.1. Let $V \in \mathcal{F}_{w}(H), W \in \mathcal{F}^{+}(H)$, and $a \in \mathbb{R}_{+}$be given, and fix $\bar{y} \in$ $\operatorname{cl}(\operatorname{Dom} A), \bar{\lambda} \in[-\infty, V(\bar{y}))$, and $\bar{\rho} \in(0,+\infty]$ so that

$$
B_{\bar{\rho}}(\bar{y}) \cap[V>\bar{\lambda}] \cap \operatorname{Dom} V \subset \operatorname{cl}(\operatorname{Dom} A) .
$$

Let $\psi: \mathbb{R} \rightarrow \mathbb{R}$ be a $\mathcal{C}^{1}$-concave function, with a positive derivative, and satisfying $\lim _{\alpha \rightarrow+\infty} \psi(\alpha)=$ 
$+\infty$. Furthermore, let assume that, for every $y \in B_{\bar{\rho}}(\bar{y}) \cap[V>\bar{\lambda}]$ and $\delta>0$,

$$
\begin{gathered}
\sup _{\xi \in \partial_{P} V(y)} \liminf _{z \rightarrow \operatorname{Dom}_{A}} \inf _{z^{*} \in A z}\left\langle\xi+\delta(y-z), f(z)-z^{*}\right\rangle+\left[\psi^{\prime}(V(y))\right]^{-1}(a V(y)+W(y)) \leq 0, \\
\sup _{\xi \in \partial_{\infty} V(y){ }_{\operatorname{Dom}_{A}} y} \liminf _{z^{*} \in A z} \inf _{z}\left\langle\xi+y-z, f(z)-z^{*}\right\rangle \leq 0 .
\end{gathered}
$$

Then, for every $x_{0} \in B_{\bar{\rho}}(\bar{y}) \cap[V>\bar{\lambda}] \cap$ Dom $V$ it holds that

$$
e^{a t} \psi\left(V\left(x\left(t ; x_{0}\right)\right)\right)+\int_{0}^{t} W\left(x\left(\tau ; x_{0}\right)\right) d \tau \leq \psi\left(V\left(x_{0}\right)\right) \quad \forall t \in\left[0, \rho\left(x_{0}\right)\right]
$$

where $\rho\left(x_{0}\right)>0$ is defined in (3.6).

Proof. First, according to Lemma 3.1 we may suppose that $W$ is Lipschitz continuous on bounded sets of $H$. Let us use the notation $\widetilde{V}:=\psi \circ V+\delta_{\text {Dom } V}$ and remark that Dom $\widetilde{V} \subset$ Dom $V$; hence, $B_{\bar{\rho}}(\bar{y}) \cap[V>\bar{\lambda}] \cap \operatorname{Dom} \widetilde{V} \subset \operatorname{cl}(\operatorname{Dom} A)$. To show that $\widetilde{V} \in \mathcal{F}_{w}(H)$, we pick a sequence $\left(y_{k}\right)_{k}$ that weakly converges to a given $y \in H$, and without any loss of generality, we may suppose that $\left(y_{k}\right)_{k} \subset \operatorname{Dom} V, y \in \operatorname{Dom} V$, and $\left(V\left(y_{k}\right)\right)_{k}$ is bounded, and therefore $\sup _{k} \psi^{\prime}\left(V\left(y_{k}\right)\right) \leq m$ for some $m \in \mathbb{R}_{+}$; otherwise, $\liminf _{k} V\left(y_{k}\right)=+\infty$ and so, since $\lim _{\alpha \rightarrow+\infty} \psi(\alpha)=+\infty$, we deduce that $\lim _{k \rightarrow \infty} \widetilde{V}\left(y_{k}\right)=\lim _{k \rightarrow \infty} \psi\left(V\left(y_{k}\right)\right)=+\infty \geq \widetilde{V}(y)$. Now, invoking the concavity of $\psi$, for each $k$ we get

$$
\widetilde{V}(y)-\widetilde{V}\left(y_{k}\right)=\psi(V(y))-\psi\left(V\left(y_{k}\right)\right) \leq \psi^{\prime}\left(V\left(y_{k}\right)\right)\left(V(y)-V\left(y_{k}\right)\right),
$$

and taking the limit as $k \rightarrow+\infty$, we obtain

$$
\widetilde{V}(y)-\liminf _{k} \widetilde{V}\left(y_{k}\right) \leq \limsup _{k} \psi^{\prime}\left(V\left(y_{k}\right)\right)\left(V(y)-V\left(y_{k}\right)\right) \leq m\left(V(y)-\liminf _{k} V\left(y_{k}\right)\right) \leq 0,
$$

showing that $\widetilde{V} \in \mathcal{F}_{w}(H)$.

The next step consists of proving that, for every given $y \in \operatorname{Dom} \widetilde{V}(\subset \operatorname{Dom} V)$,

$$
\partial_{P} \widetilde{V}(y) \subset \psi^{\prime}(V(y)) \partial_{P} V(y), \partial_{\infty} \widetilde{V}(y) \subset \partial_{\infty} V(y) .
$$

Indeed, if $\xi \in \partial_{P} \widetilde{V}(y)$, there exits $\alpha \geq 0$ such that for all $z \in$ Dom $V$ close enough to $y$ one has, using the current assumptions on $\psi$,

$$
\langle\xi, z-y\rangle \leq \psi(V(z))-\psi(V(y))+\alpha\|y-z\|^{2} \leq \psi^{\prime}(V(y))(V(z)-V(y))+\alpha\|y-z\|^{2} ;
$$

thus, $\xi \in \psi^{\prime}(V(y)) \partial_{P} V(y)$. While, for $\xi \in \partial_{\infty} \widetilde{V}(y)$ there are sequences $y_{k} \underset{\widetilde{V}}{\rightarrow} y, \alpha_{k} \rightarrow 0^{+}$, and $\xi_{k} \in \partial_{P} \widetilde{V}\left(y_{k}\right)$ such that $\alpha_{k} \xi_{k} \rightarrow \xi$; w.l.o.g., we may suppose that $\left(y_{k}\right)_{k} \subset \operatorname{Dom} \widetilde{V}(\subset \operatorname{Dom} V)$ and $\left(V\left(y_{k}\right)\right)_{k}$ is bounded (as $\left.\psi\left(V\left(y_{k}\right)\right) \rightarrow \psi\left(V\left(y_{k}\right)\right) \in \mathbb{R}\right)$. Also, subsequencing if necessary, in view of the properties of $\psi$ and the fact that $\widetilde{V}\left(y_{k}\right) \rightarrow \widetilde{V}(y)$ we may suppose that $V\left(y_{k}\right) \rightarrow V(y)$ and, so, $y_{k} \underset{V}{\rightarrow} y$ and $\psi^{\prime}\left(V\left(y_{k}\right)\right) \rightarrow \psi^{\prime}(V(y))$. Now, for each $k$, it follows from above that $\hat{\xi}_{k}:=$ $\left[\psi^{\prime}\left(V\left(y_{k}\right)\right)\right]^{-1} \xi_{k} \in \partial_{P} V\left(y_{k}\right)$. Thus, since that $\tilde{\alpha}_{k}:=\psi^{\prime}\left(V\left(y_{k}\right)\right) \alpha_{k} \rightarrow \psi^{\prime}(V(y)) 0^{+}=0^{+}, y_{k} \rightarrow \underset{V}{ } y$ and $\tilde{\alpha}_{k} \hat{\xi}_{k}=\alpha_{k} \xi_{k} \rightarrow \xi$ we deduce that $\xi \in \partial_{\infty} V(y)$. Finally, we conclude by appling Theorem $3.3($ iv) to the pair $(\widetilde{V}, W)$. 
The following result follows the lines of $[25,30]$.

Corollary 5.2. Let $V \in \mathcal{F}_{w}(H)$ satisfy $\operatorname{Dom} V \subset \operatorname{cl}(\operatorname{Dom} A), W \in \mathcal{F}^{+}(H)$, and $a \in \mathbb{R}_{+}$. We assume that, for all $y \in \operatorname{Dom} V$,

$$
\liminf _{\lambda \rightarrow 0+} \lambda^{-1}\left[V\left(J_{\lambda}(\lambda f(y)+y)\right)-V(y)\right]+a V(y)+W(y) \leq 0 .
$$

Then, $(V, W)$ is an a-Lyapunov pair for (1.1).

Proof. First, as in Corollary 5.1, w.l.o.g. $W$ is assumed to be Lipschitz continuous on bounded sets of $H$. We fix $\varepsilon>0$ and take $y \in \operatorname{Dom} V$ and $(\xi, \eta) \in \widetilde{\mathrm{N}}_{\text {epi } V}^{P}(y, V(y))$. By the current assumption, we take sequences $\left(\lambda_{k}\right)_{k}$ converging to $0^{+}$as $k \rightarrow+\infty$ and $\left(y_{k}\right)_{k}$ such that

$$
y_{k}:=J_{\lambda_{k}}\left(\lambda_{k} f(y)+y\right) \rightarrow y, \lambda_{k}^{-1}\left(y-y_{k}\right) \rightarrow y, \text { and } \lambda_{k}^{-1}\left\|y_{k}-y\right\|^{2} \rightarrow 0,
$$

together with

$$
\left(\begin{array}{c}
y_{k} \\
V(y)+\lambda_{k}[\varepsilon-a V(y)-W(y)]
\end{array}\right) \in \operatorname{epi} V \text { for } k=1,2, \cdots .
$$

Let us also set $y_{k}^{*}$ for $\lambda_{k}^{-1}\left(\lambda_{k} f(y)+y-y_{k}\right)$. Then, $y_{k}^{*} \in A y_{k}$ and

$$
\liminf _{k \rightarrow+\infty}\left\langle y-y_{k},-y_{k}^{*}\right\rangle=\liminf _{k \rightarrow+\infty}\left\langle y-y_{k},-f(y)-\lambda_{k}^{-1}\left(y-y_{k}\right)\right\rangle \leq 0 .
$$

Now, as $(\xi, \eta) \in \widetilde{\mathrm{N}}_{\text {epi } V}^{P}(y, V(y))$, from (5.1) we may suppose that, for each $k \in \mathbb{N}^{*}$,

$$
\left\langle\left(\begin{array}{c}
\xi \\
\eta
\end{array}\right),\left(\begin{array}{c}
y_{k}-y \\
\lambda_{k}[\varepsilon-a V(y)-W(y)]
\end{array}\right)\right\rangle \leq\left\|y_{k}-y\right\|^{2}+\lambda_{k}^{2}\|\varepsilon-a V(y)-W(y)\|^{2},
$$

from which we obtain

$$
\begin{aligned}
\left\langle\xi, f\left(y_{k}\right)-y_{k}^{*}\right\rangle & =\lambda_{k}^{-1}\left\langle(\xi, \eta),\left(y_{k}-y, \lambda_{k}(\varepsilon-a V(y)-W(y))\right)\right\rangle \\
& +\eta(a V(y)+W(y)-\varepsilon)+\left\langle\xi, f\left(y_{k}\right)-f(y)\right\rangle \\
& \leq \lambda_{k}^{-1}\left\|y_{k}-y\right\|^{2}+\lambda_{k}\|\varepsilon-a V(y)-W(y)\|^{2} \\
& +\eta(a V(y)+W(y)-\varepsilon)+\left\langle\xi, f\left(y_{k}\right)-f(y)\right\rangle .
\end{aligned}
$$

Hence, by the Lipschitz continuity of $f$ on $\operatorname{cl}(\operatorname{Dom} A)$, and by virtue of (5.2), we deduce that

$$
\liminf _{z \underset{\operatorname{Dom}}{\rightarrow}} \inf _{z^{*} \in A z}\left\langle\xi+y-z, f(z)-z^{*}\right\rangle \leq \liminf _{k \rightarrow+\infty}\left\langle\xi+y-y_{k}, f\left(y_{k}\right)-y_{k}^{*}\right\rangle \leq \eta(a V(y)+W(y)-\varepsilon) .
$$

Finally, when $\varepsilon \rightarrow 0^{+}$, Theorem 3.3(v) follows as well as the desired conclusion.

The following Corollary, originally given in [1], is an immediate consequence of Theorem 3.3.

Corollary 5.3. Let $\varphi \in \mathcal{F}(H)$ be convex, $V \in \mathcal{F}_{w}(H)$ satisfy $\operatorname{Dom} V \subset \operatorname{cl}(\operatorname{Dom} \varphi)$, $W \in \mathcal{F}^{+}(H)$, and $a \in \mathbb{R}_{+}$. We assume that, for all $y \in \operatorname{Dom} V$ and $\delta>0$,

$$
\sup _{\xi \in \partial_{P} V(y)} \liminf _{z \underset{\operatorname{Dom}(\partial \varphi)}{\rightarrow} y} \varphi(z-\delta(y-z)-\xi)-\varphi(z)+\langle\xi, f(y)\rangle+a V(y)+W(y) \leq 0
$$




$$
\sup _{\xi \in \partial_{\infty} V(y)} \liminf _{\substack{\operatorname{Dom}(\partial \varphi)}} \varphi(2 z-y-\xi)-\varphi(z)+\langle\xi, f(y)\rangle \leq 0
$$

Then, $(V, W)$ is an a-Lyapunov pair for (1.1) when $A=\partial \varphi$.

Proof. We take $\delta>0$ and $\xi \in \partial_{P} V(y)$ for some $y \in \operatorname{Dom} V \subset \operatorname{cl}(\operatorname{Dom} \varphi)(=\operatorname{cl}(\operatorname{Dom}(\partial \varphi)))$. If $\varepsilon>0$ is given, by the current assumption we find a sequence $\left(y_{k}, y_{k}^{*}\right)_{k} \subset \partial \varphi$ such that $\left(y_{k}\right)_{k}$ converges to $y$ and

$$
\varphi\left(y_{k}-\delta\left(y-y_{k}\right)-\xi\right)-\varphi\left(y_{k}\right)+\left\langle\xi, f\left(y_{k}\right)\right\rangle \leq \varepsilon-a V(y)-W(y) \text { for } k=1,2, \cdots .
$$

Then, for each $k \in \mathbb{N}^{*}$ we write

$$
\begin{aligned}
\left\langle\xi+\delta\left(y-y_{k}\right), f\left(y_{k}\right)-y_{k}^{*}\right\rangle & =\left\langle\xi+\delta\left(y-y_{k}\right), f\left(y_{k}\right)\right\rangle+\left\langle y_{k}-\delta\left(y-y_{k}\right)-\xi-y_{k}, y_{k}^{*}\right\rangle \\
& \leq\left\langle\xi+\delta\left(y-y_{k}\right), f\left(y_{k}\right)\right\rangle+\varphi\left(y_{k}-\delta\left(y-y_{k}\right)-\xi\right)-\varphi\left(y_{k}\right) \\
& \leq \delta\left\langle y-y_{k}, f\left(y_{k}\right)\right\rangle-a V(y)-W(y)+\varepsilon .
\end{aligned}
$$

Thus, by taking limits as $k \rightarrow+\infty$ and next letting $\varepsilon \rightarrow 0$, we obtain that

$$
\sup _{\xi \in \partial_{P} V(y)} \liminf _{z \rightarrow \underset{D o m}{\rightarrow} y} \inf _{z^{*} \in A z}\left\langle\xi+\delta(y-z), f(z)-z^{*}\right\rangle \leq-a V(y)-W(y) .
$$

Following the same argument we show that

$$
\sup _{\xi \in \partial_{\infty} V(y)} \liminf _{z \underset{\operatorname{Dom} A}{\rightarrow} y} \inf _{z^{*} \in A z}\left\langle\xi+y-z, f(z)-z^{*}\right\rangle \leq 0,
$$

and, so, the conclusion follows from Theorem 3.3(iv).

To fix ideas, let us end up the section by discussing the simple case $A=0$ of an ordinary differential equation. Then, (1.1) reads: for every $x_{0} \in H$ there exists a unique $x\left(\cdot ; x_{0}\right) \in$ $\mathcal{C}^{1}(0, \infty ; H)$ such that $x\left(0 ; x_{0}\right)=x_{0}$ and

$$
\dot{x}\left(t ; x_{0}\right)=f\left(x\left(t ; x_{0}\right)\right) \text { for all } t \geq 0 .
$$

In this case, Theorem 3.4 simplifies to (see $[20,37]$ for related results):

Corollary 5.4. Let $V \in \mathcal{F}_{w}(H)$ satisfy $\operatorname{Dom} V \subset \operatorname{cl}(\operatorname{Dom} A), W \in \mathcal{F}^{+}(H)$, and $a \in \mathbb{R}_{+}$. Then, the following statements are equivalent:

(i) $(V, W)$ is an a-Lyapunov pair for (5.3);

(ii) for every $y \in \operatorname{Dom} V$,

$$
V^{\prime}(y, f(y))+a V(y)+W(y) \leq 0 ;
$$

(iii) for every $y \in \operatorname{Dom} V$,

$$
\sup _{\xi \in \partial V(y)}\langle\xi, f(y)\rangle+a V(y)+W(y) \leq 0,
$$

where $\partial V$ either stands for $\partial_{P} V, \partial_{L} V, \partial_{F} V$, or $\partial_{C} V$.

Proof. The equivalence of (i), (ii), and ((iii) with $\left.\partial=\partial_{P}\right)$ comes from Theorem 3.4. So, we only need to prove that (iii) for $\partial=\partial_{P}$ implies (iii) for $\partial=\partial_{C}$, since $\partial_{C}$ is the largest among all the cited subdifferentials. We fix $y \in \operatorname{Dom} V$; if $\xi \in \partial_{L} V(y)$, then there exist $y_{k} \underset{V}{\rightarrow} y$ and 
$\left(\xi_{k}\right)_{k} \subset H$ such that $\xi_{k} \in \partial_{P} V\left(y_{k}\right)$ for each $k$ and $\xi_{k} \rightarrow \xi$. Thus, applying (iii) for $\partial=\partial_{P}$ yields

$$
\left\langle\xi_{k}, f\left(y_{k}\right)\right\rangle+a V\left(y_{k}\right)+W\left(y_{k}\right) \leq 0 \text { for each } k \in \mathbb{N} .
$$

Passing to the limit as $k$ goes to $+\infty$, and taking into account the lower semicontinuity of $V$ and $W$, it follows that

$$
\langle\xi, f(y)\rangle+a V(y)+W(y) \leq 0 .
$$

Now, if $\xi \in \partial_{\infty} V(y)$, then there exist $y_{k} \underset{V}{\rightarrow} y$ and $\alpha_{k} \rightarrow 0^{+}$, together with $\left(\xi_{k}\right)_{k} \subset H$, such that $\xi_{k} \in \partial_{P} V\left(y_{k}\right)$ for each $k$ and $\alpha_{k} \xi_{k} \rightarrow \xi$. Thus, $\xi_{k}$ and $y_{k}$ satisfy (5.4) which in turn, after multiplying by $\alpha_{k}$ and next taking limits as $k \rightarrow+\infty$, gives us $\langle\xi, f(y)\rangle \leq 0$. Consequently, (iii) for $\partial=\partial_{C}$ follows from the representation formula (2.3): $\partial_{C} V(y)=\overline{\mathrm{co}}\left\{\partial_{L} V(y) \cup \partial_{\infty} V(y)\right\}$.

6. Appendix: proof of Theorem $3.3((\mathrm{v}) \Longrightarrow(\mathrm{i}))$ and Theorem $3.4((\mathrm{iv}) \Longrightarrow(\mathrm{i}))$. This section is devoted to complete the missing proofs of Theorems 3.3 and 3.4.

We begin by giving a couple of lemmas:

Lemma 6.1. Let $S \subset H$ be a weakly closed set. Then, for every $x \in H \backslash S$ we have that

$$
\partial_{L} d(\cdot, S)(x) \subset\left\{\frac{x-\Pi_{S}(x)}{d(x, S)}\right\} .
$$

Proof. We fix $x \in H \backslash S$ and pick $\xi \in \partial_{L} d(\cdot, S)(x)$. Then, there exist sequences $\left(x_{k}\right)_{k},\left(\xi_{k}\right)_{k} \subset H$ such that $x_{k} \rightarrow x, \xi_{k} \in \partial_{P} d\left(x_{k}, S\right)$ for every $k$, and $\xi_{k} \rightarrow \xi$. In particular, for each $k$, we may suppose that $x_{k} \notin S$ and so $\left\{\Pi_{S}\left(x_{k}\right)\right\}$ is a singleton and $\xi_{k}=\frac{x_{k}-\Pi_{S}\left(x_{k}\right)}{d\left(x_{k}, S\right)}$ (see, e.g., [21]). Furthermore, as $\left\|x_{k}-\Pi_{S}\left(x_{k}\right)\right\| \leq d\left(x_{k}, S\right) \leq\left\|x_{k}-x\right\|+d(x, S)$, the sequence $\left(\Pi_{S}\left(x_{k}\right)\right)_{k}$ is bounded and so, subsequencing if necessary, we may assume that it weakly converges, say to some $\bar{x} \in S$. Hence, writing

$$
\|x-\bar{x}\| \leq \liminf _{k \rightarrow+\infty}\left\|x_{k}-\Pi_{S}\left(x_{k}\right)\right\|=\lim _{k} d\left(x_{k}, S\right)=d(x, S),
$$

it follows that $\bar{x} \in \Pi_{S}(x)$ and, so,

$$
\xi=\text { weak }-\lim _{k} \xi_{k}=\lim _{k} \xi_{k}=\frac{x-\bar{x}}{d(x, S)} \subset \frac{x-\Pi_{S}(x)}{d(x, S)},
$$

as we wanted to prove.

The following lemma is the Gronwall Lemma (e.g., [1, Lemma 1]).

Lemma 6.2. Given $t_{2}>t_{1} \geq 0, a \neq 0$, and $b \geq 0$, we assume that the absolutely continuous function $h:\left[t_{1}, t_{2}\right] \rightarrow \mathbb{R}_{+}$satisfies $h^{\prime}(t) \leq a h(t)+b$ a.e. $t \in\left[t_{1}, t_{2}\right]$. Then, for all $t \in\left[t_{1}, t_{2}\right]$,

$$
h(t) \leq\left(h\left(t_{1}\right)+\frac{b}{a}\right) e^{a\left(t-t_{1}\right)}-\frac{b}{a} .
$$

6.1. Proof of Theorem $3.3((\mathbf{v}) \Longrightarrow(\mathrm{i}))$. Proof. First, according to Lemma 3.1 we may assume (w.l.o.g.) that $W$ is Lipschitz continuous on every compact subset of $H$; hence, we shall use $L_{W}$ to refer to its Lipschitz constant on $\bar{B}_{\bar{\rho}}(\bar{y})$. We recall that $L_{f}$ denotes the Lipschitz constant of the mapping $f$ on $\operatorname{cl}(\operatorname{Dom} A)$.

In this first part we gather the notation that we will use in the remainder of the proof. We 
fix $x_{0} \in B_{\bar{\rho}}(\bar{y}) \cap[V>\bar{\lambda}] \cap$ Dom $V$ and according to the weak lower semicontinuity of $V$ we fix $\rho>0$ such that

$$
B_{\rho}\left(x_{0}\right) \subset B_{\bar{\rho}}(\bar{y}) \cap[V>\bar{\lambda}] .
$$

Using the continuity of $x\left(\cdot ; x_{0}\right)$, we also select $\nu>0$ such that

$$
\sup _{t \in[0, \nu]} 2\left\|x\left(t ; x_{0}\right)-x_{0}\right\|+\left|\left(e^{-a t}-1\right) V\left(x_{0}\right)-\int_{0}^{t} W\left(x\left(\tau ; x_{0}\right)\right) d \tau\right|<\rho .
$$

Next, we fix $T>\nu$ and take a sequence $\left(y_{n}\right)_{n \in \mathbb{N}^{*}} \subset$ Dom $A$ that converges to $x_{0}$. Let $x_{n}\left(\cdot ; y_{n}\right)$ denote the (strong) solution of the differential inclusion

$$
\dot{x}_{n}\left(t ; y_{n}\right) \in f\left(x_{n}\left(t ; y_{n}\right)\right)-A x_{n}\left(t ; y_{n}\right) \text {, a.e. } t \geq 0, x_{n}\left(0, y_{n}\right)=y_{n} .
$$

We know that the sequence $\left(x_{n}\left(\cdot ; y_{n}\right)\right)_{n}$ uniformly converges to $x\left(\cdot ; x_{0}\right)$ on $[0, T]$ and, so, we may suppose that (see (6.1) and (6.2)), for all $t \in[0, \nu]$ and every $n, n^{\prime} \geq 1$,

$$
\begin{gathered}
x_{n}\left(t ; y_{n}\right) \in B_{\frac{\rho}{2}}\left(x_{0}\right)\left(\subset B_{\bar{\rho}}(\bar{y})\right) \\
2\left\|x_{n^{\prime}}\left(t ; y_{n^{\prime}}\right)-x_{0}\right\|+\left|\left(e^{-a t}-1\right) V\left(x_{0}\right)-\int_{0}^{t} W\left(x_{n}\left(\tau ; y_{n}\right)\right) d \tau\right|<\rho .
\end{gathered}
$$

Now, if $n \in \mathbb{N}^{*}$ is fixed, we define the $\mathcal{C}^{1}$-function $h_{n}:[0, T] \subset \mathbb{R}_{+} \rightarrow \mathbb{R}_{+}$as

$$
h_{n}(t):=\int_{0}^{t} W\left(x_{n}\left(\tau ; y_{n}\right)\right) d \tau ;
$$

and the functions $\gamma_{n}(\cdot):[0, T] \subset \mathbb{R}_{+} \rightarrow \mathbb{R}, z_{n}(\cdot):[0, T] \subset \mathbb{R}_{+} \rightarrow H \times \mathbb{R}$, and $\eta_{n}:[0, T] \subset \mathbb{R}_{+} \rightarrow$ $\mathbb{R}_{+}$given respectively by

$$
\gamma_{n}(t):=e^{-a t}\left(V\left(x_{0}\right)-h_{n}(t)\right), z_{n}(t):=\left(x_{n}\left(t ; y_{n}\right), \gamma_{n}(t)\right), \eta_{n}(t):=\frac{1}{2} d^{2}\left(z_{n}(t), \text { epi } V\right) .
$$

We observe that each function $\eta_{n}$ is Lipschitz continuous on every compact interval in $(0, T]$ so that, using a well known chain rule (e.g. [19]), for all $t \in(0, T)$ we obtain

$$
\partial_{C} \eta_{n}(t)=d\left(z_{n}(t), \text { epi } V\right) \partial_{C} d\left(z_{n}(\cdot), \text { epi } V\right)(t) \neq \emptyset .
$$

Thus we observe that if $z_{n}(t) \in$ epi $V$, then $\partial_{C} \eta_{n}(t)=\{0\}$.

In order to establish Theorem 3.3(i), we shall proceed by step.

First, in the following lemma we give a general estimation of $\partial_{C} \eta_{k}$ :

Lemma 6.3. We let $n \in \mathbb{N}^{*}$ and $t \in(0, T)$ be such that $z_{n}(t) \notin$ epi $V$. Then, we have that

$$
\partial_{C} \eta_{n}(t) \subset \overline{\mathrm{Co}}\left[\bigcup_{(u, \mu) \in \mathcal{E}}\left\langle z_{n}(t)-\left(\begin{array}{c}
u \\
\mu
\end{array}\right),\left(\begin{array}{c}
f\left(x_{n}\left(t ; y_{n}\right)\right)-A x_{n}\left(t ; y_{n}\right) \\
-a \gamma_{n}(t)-e^{-a t} W\left(x_{n}\left(t ; y_{n}\right)\right)
\end{array}\right)\right\rangle\right],
$$


where

$$
\mathcal{E}:=\Pi_{\text {epi } V}\left(z_{n}(t)\right) \cap\left(B_{\rho}\left(x_{0}\right) \times\left[\gamma_{n}(t), \gamma_{n}(t)+\rho\right]\right)
$$

Proof. We take $\xi \in d\left(z_{n}(t)\right.$, epi $\left.V\right) \partial_{L} d(\cdot$, epi $V)\left(z_{n}(t)\right)$. Since that epi $V$ is weakly closed in $H \times \mathbb{R}$, by Lemma 6.1 there exists some $(u, \mu) \in \Pi_{\text {epi } V}\left(z_{n}(t)\right)$ such that $\xi=z_{n}(t)-(u, \mu)$, and, as $\left(x_{0}, V\left(x_{0}\right)+\left(1-e^{-a t}\right) h_{n}(t)\right) \in \operatorname{epi} V$ (we recall that $\left.W \in \mathcal{F}^{+}(H)\right)$,

$$
\begin{aligned}
\max \left\{\left|\mu-\gamma_{n}(t)\right|,\left\|u-x_{0}\right\|\right\} & \leq\left\|(u, \mu)-z_{n}(t)\right\|+\left\|x_{n}\left(t ; y_{n}\right)-x_{0}\right\| \\
& \leq\left\|z_{n}(t)-\left(x_{0}, V\left(x_{0}\right)+\left(1-e^{-a t}\right) h_{n}(t)\right)\right\|+\left\|x_{n}\left(t ; y_{n}\right)-x_{0}\right\|
\end{aligned}
$$

Thus, by (6.2),

$$
\max \left\{\left|\mu-\gamma_{n}(t)\right|,\left\|u-x_{0}\right\|\right\} \leq 2\left\|x_{n}\left(t ; y_{n}\right)-x_{0}\right\|+\left|\left(e^{-a t}-1\right) V\left(x_{0}\right)-h_{n}(t)\right|<\rho .
$$

We also observe that $V(u)>\gamma_{n}(t)$ because, otherwise, we would have

$$
V(u) \leq \gamma_{n}(t)<V\left(x_{n}\left(t ; y_{n}\right)\right)
$$

which in turn yields the contradiction

$$
d\left(z_{n}(t) \text {, epi } V\right)=\left\|z_{n}(t)-\left(u, \gamma_{n}(t)\right)\right\|<\left\|(u, \mu)-z_{n}(t)\right\|=d\left(z_{n}(t) \text {, epi } V\right) .
$$

Consequently, we write

$$
(u, \mu) \in \Pi_{\mathrm{epi} V}\left(z_{n}(t)\right) \cap\left(B_{\rho}\left(x_{0}\right) \times\left[\gamma_{n}(t), \gamma_{n}(t)+\rho\right]\right),
$$

and so, from one hand, by (2.3) it follows that (see, e.g., [19])

$$
d\left(z_{n}(t), \text { epi } V\right) \partial_{C} d\left(z_{n}(\cdot), \text { epi } V\right)(t) \subset \overline{\mathrm{co}}\left[\bigcup_{(u, \mu) \in \mathcal{E}} \partial_{C}\left\langle z_{n}(t)-(u, \mu), z_{n}(\cdot)\right\rangle(t)\right] .
$$

On the other hand, set $D:=\left\{t \in[0, T] \mid x_{n}\left(\cdot ; y_{n}\right)\right.$ is differentiable at $t$ for all $\left.n\right\}$; hence, the set $H \backslash D$ is of measure zero, for each $n \in \mathbb{N}^{*}$ there exists a number $\delta_{n}>0$ such that

$$
\sup _{t \in D}\left\|\dot{x}_{n}\left(t ; y_{n}\right)\right\|+\left|\dot{\gamma}_{n}(t)\right| \leq \delta_{n}
$$

and, by the Lipschitz continuity of $z_{n}(\cdot)$,

$$
\partial_{C}\left\langle p^{*}, z_{n}(\cdot)\right\rangle(t)=\overline{\mathrm{co}}\left\{\lim _{n}\left\langle p^{*}, \dot{z}_{n}\left(t_{k}\right) \cap B_{\delta_{n}}\right\rangle \mid t_{k} \rightarrow t ; t_{k} \in D\right\} .
$$

Therefore, invoking the $(\|\cdot\| \times$ weak $)$ upper semicontinuity of $A$, and taking limits as $k \rightarrow+\infty$ one obtains:

$$
\partial_{C}\left\langle p^{*}, z_{n}(\cdot)\right\rangle(t) \subset\left\{\left\langle p^{*},\left(\begin{array}{c}
f\left(x_{n}\left(t ; y_{n}\right)\right)-A x_{n}\left(t ; y_{n}\right) \\
-a \gamma_{n}(t)-e^{-a t} h_{n}^{\prime}(t)
\end{array}\right)\right\rangle\right\} .
$$

Combining (6.11) to (6.10) yields the desired conclusion of the current lemma.

Next, we improve the formula given in Lemma 6.3, provided that the current assumption 
(Theorem 3.3(v)) is assumed.

Lemma 6.4. We let $n \in \mathbb{N}^{*}$ and $t \in(0, T)$ be given. Then, the following inclusion holds:

$$
\partial_{C} \eta_{n}(t) \subset\left(-\infty, 2\left(L_{f}+L_{W}\right) \eta_{n}(t)\right] \text { for all } t \in[0, \nu] .
$$

Proof. We suppose that $V\left(x_{n}\left(t ; y_{n}\right)\right)>\gamma_{n}(t)$ because, otherwise, $\partial_{C} \eta_{n}(t)=\{0\}$ and the conclusion obviously holds. Then, according to Lemma 6.3, it suffices to show that for every fixed $x_{n}^{*} \in A x_{n}\left(t ; y_{n}\right)$ and $(u, \mu) \in \Pi_{\text {epi } V}\left(z_{n}(t)\right) \cap\left(B_{\rho}\left(x_{0}\right) \times\left[\gamma_{n}(t), \gamma_{n}(t)+\rho\right]\right)$ it holds that

$$
\psi\left(u, \mu, x_{n}^{*}\right):=\left\langle z_{n}(t)-\left(\begin{array}{c}
u \\
\mu
\end{array}\right),\left(\begin{array}{c}
f\left(x_{n}\left(t ; y_{n}\right)\right)-x_{n}^{*} \\
-a \gamma_{n}(t)-e^{-a t} W\left(x_{n}\left(t ; y_{n}\right)\right)
\end{array}\right)\right\rangle \leq 2\left(L_{f}+L_{W}\right) \eta_{n}(t) .
$$

Indeed, as the vector $(u, \mu)\left(\in \Pi_{\text {epi } V}\left(z_{n}(t)\right)\right)$ obviously verifies

$$
\left\langle z_{n}(t)-(u, \mu),\left(u^{\prime}, \mu^{\prime}\right)-(u, \mu)\right\rangle \leq\left\|\left(u^{\prime}, \mu^{\prime}\right)-(u, \mu)\right\|^{2} \quad \forall\left(u^{\prime}, \mu^{\prime}\right) \in \text { epi } V,
$$

we have that $z_{n}(t)-(u, \mu) \in \widetilde{\mathrm{N}}_{\text {epi } V}^{P}(u, \mu)$ (remember $\left.(2.5)\right)$, with $u \in B_{\rho}\left(x_{0}\right) \cap \operatorname{Dom} V \subset$ $\operatorname{cl}($ Dom $A) \cap B_{\bar{\rho}}(\bar{y}) \cap[V>\bar{\lambda}]$, according to (6.1). Hence, taking into account the current assumption (Theorem $3.3(\mathrm{v})$ ), there exists a sequence $\left(u_{k}, u_{k}^{*}\right)_{k} \subset A$ such that $u_{k} \rightarrow u$ and, for all $k \geq 1$,

$$
\begin{gathered}
L_{f}\left\|x_{n}\left(t ; y_{n}\right)-u\right\|\left\|u-u_{k}\right\|+\left\|u_{k}-u\right\|\left\|f\left(u_{k}\right)-x_{n}^{*}\right\| \leq \frac{1}{k}, \\
\left\langle x_{n}\left(t ; y_{n}\right)-u_{k}, f\left(u_{k}\right)-u_{k}^{*}\right\rangle \leq\left(\gamma_{n}(t)-\mu\right)(a V(u)+W(u))+\frac{1}{k} .
\end{gathered}
$$

Thus, writing

$$
\begin{aligned}
\left\langle x_{n}\left(t ; y_{n}\right)-u, f\left(x_{n}\left(t ; y_{n}\right)\right)-x_{n}^{*}\right\rangle= & \left\langle x_{n}\left(t ; y_{n}\right)-u, f\left(x_{n}\left(t ; y_{n}\right)\right)-f\left(u_{k}\right)\right\rangle \\
& +\left\langle x_{n}\left(t ; y_{n}\right)-u_{k}, f\left(u_{k}\right)-u_{k}^{*}\right\rangle \\
& +\left\langle x_{n}\left(t ; y_{n}\right)-u_{k}, u_{k}^{*}-x_{n}^{*}\right\rangle \quad(\leq 0 \text { as } A \text { is monotone }) \\
& +\left\langle u_{k}-u, f\left(u_{k}\right)-x_{n}^{*}\right\rangle,
\end{aligned}
$$

and using the $L_{f}$-Lipschitz continuity of $f$ yields

$$
\begin{aligned}
\left\langle x_{n}\left(t ; y_{n}\right)-u, f\left(x_{n}\left(t ; y_{n}\right)\right)-x_{n}^{*}\right\rangle & \leq L_{f}\left\|x_{n}\left(t ; y_{n}\right)-u\right\|\left\|x_{n}\left(t ; y_{n}\right)-u_{k}\right\| \\
& +\left(\gamma_{n}(t)-\mu\right)(a V(u)+W(u))+\frac{1}{k} \\
& +\left\|u_{k}-u\right\|\left\|f\left(u_{k}\right)-x_{n}^{*}\right\| \\
& \leq 2 L_{f} \eta_{n}(t)+\left(\gamma_{n}(t)-\mu\right)(a V(u)+W(u))+\frac{2}{k},
\end{aligned}
$$


where in the last inequality we have used (6.14). Consequently, when $k \rightarrow+\infty$,

$$
\begin{aligned}
\psi\left(u, \mu, x_{n}^{*}\right) & =\left\langle x_{n}\left(t ; y_{n}\right)-u, f\left(x_{n}\left(t ; y_{n}\right)\right)-x_{n}^{*}\right\rangle \\
& +a\left(\mu-\gamma_{n}(t)\right) \gamma_{n}(t)+\left(\mu-\gamma_{n}(t)\right) e^{-a t} W\left(x_{n}\left(t ; x_{n}\right)\right) \\
& \leq 2 L_{f} \eta_{n}(t)-\left(\mu-\gamma_{n}(t)\right)(a V(u)+W(u)) \\
& +a\left(\mu-\gamma_{n}(t)\right) \gamma_{n}(t)+\left(\mu-\gamma_{n}(t)\right) e^{-a t} W\left(x_{n}\left(t ; y_{n}\right)\right) \\
& =a\left(\mu-\gamma_{n}(t)\right)\left(\gamma_{n}(t)-V(u)\right) \\
& +2 L_{f} \eta_{n}(t)+\left(\mu-\gamma_{n}(t)\right)\left(e^{-a t} W\left(x_{n}\left(t ; y_{n}\right)\right)-W(u)\right) \\
& \leq 2 L_{f} \eta_{n}(t)+\left(\mu-\gamma_{n}(t)\right) e^{-a t}\left(W\left(x_{n}\left(t ; y_{n}\right)\right)-W(u)\right) .
\end{aligned}
$$

But, $u, x_{n}\left(t ; y_{n}\right) \in B_{\rho}\left(x_{0}\right) \subset B_{\bar{\rho}}(\bar{y})$ and $W$ is $L_{W}$-Lipschitz continuous on $\bar{B}_{\bar{\rho}}(\bar{y})$, and so

$$
\psi\left(u, \mu, x_{n}^{*}\right) \leq 2 L_{f} \eta_{n}(t)+L_{W}\left|\mu-\gamma_{n}(t)\right|\left\|x_{n}\left(t ; y_{n}\right)-u\right\| \leq 2\left(L_{f}+L_{W}\right) \eta_{n}(t),
$$

establishing (6.13).

Finally, in the last lemma we obtain the desired conclusion.

LEMMA 6.5. Theorem 3.4(i) holds; that is, for all $t \in\left[0, \rho\left(x_{0}\right)\right]$ we have that

$$
e^{a t} V\left(x\left(t ; x_{0}\right)\right)+\int_{0}^{t} W\left(x\left(\tau ; x_{0}\right)\right) d \tau \leq V\left(x_{0}\right)
$$

Proof. As the number $\nu$ can be chosen close enough to $\rho\left(x_{0}\right)$ (remember $(6.2)$ ), in view of the weak lower semicontinuity of $V$ and the continuity of $x\left(\cdot ; x_{0}\right)$ it suffices to show that (6.15) holds on $[0, \nu]$. For this aim, we fix $t_{1}, t_{2} \in(0, \nu), t \in\left(t_{1}, t_{2}\right)$, and $n \in \mathbb{N}^{*}$. By invoking (6.12) together with Lemma 6.2, we obtain that

$$
e^{-2\left(L_{f}+L_{W}\right) t}\left(L_{f}+L_{W}\right) \eta_{n}(t) \leq e^{-2\left(L_{f}+L_{W}\right) t_{1}}\left(L_{f}+L_{W}\right) \eta_{n}\left(t_{1}\right)
$$

Thus, by making $t_{1} \rightarrow 0$, it follows that $d\left(\left(x_{n}\left(t ; y_{n}\right), e^{-a t}\left(V\left(x_{0}\right)-h_{n}(t)\right)\right)\right.$, epi $\left.V\right)=0$ which in turn gives us, as $n \rightarrow+\infty$,

$$
e^{a t} V\left(x\left(t ; x_{0}\right)\right)+\int_{0}^{t} W\left(x\left(\tau ; x_{0}\right)\right) d \tau \leq V\left(x_{0}\right)
$$

as we wanted to prove.

6.2. Proof of Theorem 3.4 ((iv) $\Longrightarrow($ i)). Proof. Let us recall the notation used in the proof of Theorem $3.3\left((\mathrm{v}) \Longrightarrow(\mathrm{i})\right.$ ) (see Page 26): for a fixed $x_{0} \in B_{\bar{\rho}}(\bar{y}) \cap[V>\bar{\lambda}] \cap \operatorname{Dom} V$, the numbers $\rho, \nu>0$ satisfy (6.1) and (6.2). For given $T>\nu$ and $\left(y_{n}\right)_{n \in \mathbb{N}^{*}} \subset \operatorname{Dom} A, x_{n}\left(\cdot ; y_{n}\right)$ is the strong solution of $(6.3)$ so that $(6.4)$ and $(6.5)$ hold, and $x_{n}\left(\cdot ; y_{n}\right)$ uniformly converges to $x\left(\cdot ; x_{0}\right)$ on $[0, T]$. As well, the functions $h_{n}, \gamma_{n}(\cdot), z_{n}(\cdot), \eta_{n}(t), n \in \mathbb{N}^{*}$, are those defined in (6.6) and (6.7).

As in the proof of Theorem 3.3, namely, Lemma 6.5, it suffices to show that the current 
assumption (iv) leads us, for every given $n \in \mathbb{N}^{*}$ and $t \in(0, T)$ such that $z_{n}(t) \notin$ epi $V$, to

$$
\partial_{C} \eta_{n}(t) \subset\left(-\infty, 2\left(L_{W}+L_{f}\right) \eta_{n}(t)\right] \text { for all } t \in[0, \nu]
$$

But, we know that (see (6.10) and (6.11))

$$
\partial_{C} \eta_{n}(t) \subset \overline{\mathrm{co}}\left[\bigcup_{(u, \mu) \in d\left(z_{n}(t), \mathrm{epi} V\right) \partial_{L} d(\cdot, \mathrm{epi} V)\left(z_{n}(t)\right)}\left\langle(u, \mu),\left(\begin{array}{c}
f\left(x_{n}\left(t ; y_{n}\right)\right)-A x_{n}\left(t ; y_{n}\right) \\
-a \gamma_{n}(t)-e^{-a t} W\left(x_{n}\left(t ; y_{n}\right)\right)
\end{array}\right)\right\rangle,\right.
$$

and so we will be done if we show that, for every given $(u, \mu) \in d\left(z_{n}(t)\right.$, epi $\left.V\right) \partial_{L} d(\cdot$, epi $V)\left(z_{n}(t)\right)$ and $x_{n}^{*} \in A x_{n}\left(t ; y_{n}\right)$,

$$
\left\langle(u, \mu),\left(\begin{array}{c}
f\left(x_{n}\left(t ; y_{n}\right)\right)-x_{n}^{*} \\
-a \gamma_{n}(t)-e^{-a t} W\left(x_{n}\left(t ; y_{n}\right)\right)
\end{array}\right)\right\rangle \leq 2\left(L_{W}+L_{f}\right) \eta_{n}(t)
$$

In this respect, by the definition of $\partial_{L}$ we select sequences $\left(y_{k}, \gamma_{k}\right)_{k},\left(u_{k}, \mu_{k}\right)_{k} \subset H \times \mathbb{R}$ be such that (see the proof of Lemma 6.1)

$$
\left(y_{k}, \gamma_{k}\right)-\left(u_{k}, \mu_{k}\right) \rightarrow(u, \mu),\left(y_{k}, \gamma_{k}\right) \rightarrow z_{n}(t),\left\{\left(u_{k}, \mu_{k}\right)\right\}=\Pi_{\mathrm{epi} V}\left(y_{k}, \gamma_{k}\right) \quad \forall k \geq 1
$$

hence,

$$
\left(y_{k}, \gamma_{k}\right)-\left(u_{k}, \mu_{k}\right) \in \mathrm{N}_{\text {epi } V}^{P}\left(u_{k}, \mu_{k}\right), \gamma_{k} \leq \mu_{k}, V\left(u_{k}\right)=\mu_{k}
$$

Furthermore, arguing as in (6.8) and using the assumption on the operator $A$, we can assume that

$$
u_{k} \in B_{\rho}\left(x_{0}\right) \cap \operatorname{Dom} V \subset B_{\bar{\rho}}(\bar{y}) \cap[V>\bar{\lambda}] \cap \operatorname{Int}(\operatorname{Dom} A) \quad \forall k \geq 1 ;
$$

that is, w.l.o.g., $\left(u_{k}\right)_{k}$ weakly converges to some $\bar{u} \in B_{\rho}\left(x_{0}\right)$. So, taking into account the weak lower semicontinuity of $V$, together with the current assumption (iv), we get that $\bar{u} \in B_{\rho}\left(x_{0}\right) \cap$ Dom $V \subset B_{\bar{\rho}}(\bar{y}) \cap[V>\bar{\lambda}] \cap \operatorname{Int}(\operatorname{Dom} A)$. In other words, using again the maximal monotonicity of $A$, we may suppose that the sets $\left\{A u_{k}\right\}, k \geq 1$, are uniformly bounded.

Now, we fix $\varepsilon>0$. If $\mu_{k}-\gamma_{k}>0$ for infinitely many $k,(6.17) \operatorname{reads}\left(\mu_{k}-\gamma_{k}\right)^{-1}\left(y_{k}-u_{k}\right) \in$ $\partial_{P} V\left(u_{k}\right)$ so that by the current assumption we find $u_{k}^{*} \in A u_{k}$ such that

$$
\left\langle\left(\mu_{k}-\gamma_{k}\right)^{-1}\left(y_{k}-u_{k}\right), f\left(u_{k}\right)-u_{k}^{*}\right\rangle+a V\left(u_{k}\right)+W\left(u_{k}\right) \leq \varepsilon\left(\mu_{k}-\gamma_{k}\right)^{-1} ;
$$

moreover, as the sets $\left\{A u_{k}\right\}$ are bounded independently of $k$, we may assume that (w.l.o.g.)

$$
\left\langle y_{k}-x_{n}\left(t ; y_{n}\right), u_{k}^{*}-x_{n}^{*}\right\rangle \leq \varepsilon \text { for all } k \geq 1
$$

Then, we write

$$
\begin{array}{rlll}
\left\langle y_{k}-u_{k}, f\left(y_{k}\right)-x_{n}^{*}\right\rangle & =\left\langle y_{k}-u_{k}, f\left(y_{k}\right)-f\left(u_{k}\right)\right\rangle & & \left(\leq L_{f}\left\|y_{k}-u_{k}\right\|^{2}\right) \\
& +\left\langle y_{k}-x_{n}\left(t ; y_{n}\right), u_{k}^{*}-x_{n}^{*}\right\rangle & (\leq \varepsilon) \\
& +\left\langle x_{n}\left(t ; y_{n}\right)-u_{k}, u_{k}^{*}-x_{n}^{*}\right\rangle & & (\leq 0 \text { by the monotonicity of } A) \\
& +\left\langle y_{k}-u_{k}, f\left(u_{k}\right)-u_{k}^{*}\right\rangle, &
\end{array}
$$


and so, by (6.18),

$$
\left\langle y_{k}-u_{k}, f\left(y_{k}\right)-x_{n}^{*}\right\rangle \leq L_{f}\left\|y_{k}-u_{k}\right\|^{2}-\left(\mu_{k}-\gamma_{k}\right)\left(a V\left(u_{k}\right)+W\left(u_{k}\right)\right)+2 \varepsilon .
$$

Hence, recalling that $V\left(u_{k}\right)=\mu_{k} \geq \gamma_{k}$ (by (6.17)) and $W \geq 0$, we obtain that

$$
\begin{aligned}
& \left\langle\left(\begin{array}{c}
y_{k} \\
\gamma_{k}
\end{array}\right)-\left(\begin{array}{c}
u_{k} \\
\mu_{k}
\end{array}\right),\left(\begin{array}{c}
f\left(y_{k}\right)-x_{n}^{*} \\
-a \gamma_{k}-e^{-a t} W\left(y_{k}\right)
\end{array}\right)\right\rangle \\
& \quad=\left\langle y_{k}-u_{k}, f\left(y_{k}\right)-x_{n}^{*}\right\rangle+\left(\mu_{k}-\gamma_{k}\right)\left(a \gamma_{k}+e^{-a t} W\left(y_{k}\right)\right) \\
& \quad \leq L_{f}\left\|y_{k}-u_{k}\right\|^{2}-\left(\mu_{k}-\gamma_{k}\right)\left(a V\left(u_{k}\right)+W\left(u_{k}\right)\right)+\left(\mu_{k}-\gamma_{k}\right)\left(a V\left(u_{k}\right)+W\left(y_{k}\right)\right)+2 \varepsilon \\
& \quad=L_{f}\left\|y_{k}-u_{k}\right\|^{2}+\left(\mu_{k}-\gamma_{k}\right)\left(W\left(y_{k}\right)-W\left(u_{k}\right)\right)+2 \varepsilon .
\end{aligned}
$$

But, $u_{k}, y_{k} \in B_{\rho}\left(x_{0}\right) \subset B_{\bar{\rho}}(\bar{y})$ (remember that $y_{k} \rightarrow x_{n}\left(t ; y_{n}\right) \in B_{\rho}\left(x_{0}\right)$ ), and so using the $L_{W}$-Lipschitzianity of $W$, the last inequality above gives us

$$
\left\langle\left(\begin{array}{c}
y_{k} \\
\gamma_{k}
\end{array}\right)-\left(\begin{array}{c}
u_{k} \\
\mu_{k}
\end{array}\right),\left(\begin{array}{c}
f\left(y_{k}\right)-x_{n}^{*} \\
-a \gamma_{k}-e^{-a t} W\left(y_{k}\right)
\end{array}\right)\right\rangle \leq L_{f}\left\|y_{k}-u_{k}\right\|^{2}+\frac{L_{W}}{2}\left(\left|\mu_{k}-\gamma_{k}\right|^{2}+\left\|y_{k}-u_{k}\right\|^{2}\right)+2 \varepsilon ;
$$

that is, by taking the limits $k \rightarrow+\infty$ and next $\varepsilon \rightarrow 0$,

$$
\left\langle(u, \mu),\left(\begin{array}{c}
f\left(x_{n}\left(t ; y_{n}\right)\right)-x_{n}^{*} \\
-a e^{-a t}\left(V\left(x_{0}\right)-h_{n}(t)\right)-e^{-a t} W\left(x_{n}\left(t ; y_{n}\right)\right)
\end{array}\right)\right\rangle \leq 2\left(L_{W}+L_{f}\right) \eta_{n}(t)
$$

establishing (6.16).

Now, if $\mu_{k}-\gamma_{k}(t)=0$ for all $k,(6.17)$ reads $y_{k}-u_{k} \in \partial_{\infty} V\left(u_{k}\right)$ and so by the current assumption we select $u_{k}^{*} \in A u_{k}$ such that

$$
\left\langle y_{k}-u_{k}, f\left(u_{k}\right)-u_{k}^{*}\right\rangle \leq \varepsilon
$$

Moreover, as above we may assume that $\left\langle y_{k}-x_{n}\left(t ; y_{n}\right), u_{k}^{*}-x_{n}^{*}\right\rangle \leq \varepsilon$, for all $k \geq 1$. Therefore the $L_{f}$-Lipschitzianity of $f$ together with the monotonicity of $A$, imply the relation

$$
\left\langle\left(\begin{array}{c}
y_{k} \\
\gamma_{k}
\end{array}\right)-\left(\begin{array}{c}
u_{k} \\
\mu_{k}
\end{array}\right),\left(\begin{array}{c}
f\left(y_{k}\right)-x_{n}^{*} \\
-a \gamma_{k}-e^{-a t} W\left(y_{k}\right)
\end{array}\right)\right\rangle=\left\langle y_{k}-u_{k}, f\left(y_{k}\right)-x_{n}^{*}\right\rangle \leq L_{f}\left\|y_{k}-u_{k}\right\|^{2}+2 \varepsilon,
$$

from which we derive (6.16) by passing to the limit, first as $k \rightarrow+\infty$ and then as $\varepsilon \rightarrow 0$.

Acknowledgement The authors are indebted to H. Attouch, J.-F. Jaber, N. Igbida, T. Roubicek, and J. Rossi for valuable discussions on this work.

\section{REFERENCES}

[1] Adly, S., Goeleven, D. A stability theory for second-order nonsmooth dynamical systems with application to friction problems. J. Mathématiques Pures et Appliquées, 83 (2004), 17-51.

[2] Aubin, J.-P, Viability theory. Systems \& Control: Foundations \& Applications. Birkhäuser Boston, Inc., Boston, MA, 1991.

[3] Aubin, J.-P, Cellina, A. Differential inclusions. Set-valued maps and viability theory. Springer-Verlag, Berlin, 1984.

[4] Attouch, H., Baillon, J.-B. And ThÉra, M. Variational sum of monotone operators. J. Convex Anal. 1 (1994), 1-29.

[5] Bacciotti, A. Stability in the continuous case. J. Math. Anal. Appl. 270 (2002), no. 2, 488-498. 
[6] Bacciotti, A., Ceragioli, F. Stability and stabilization of discontinuous systems and nonsmooth Lyapunov functions. ESIAM: Control Optim. Calculus Variations, 4, (1999), 361-376.

[7] Bacciotti, A., Ceragioli, F. And Mazzi, L. Differential inclusions and monotonicity conditions for nonsmooth Lyapunov functions. Set-Valued Anal. 8 (2000), no. 3, 299-309.

[8] Barbu, V., Pavel, N. H. Flow-invariant closed sets with respect to nonlinear semigroup flows. NoDEA: Nonlinear Differential Equations Appl. 10 (2003), no. 1, 57-72.

[9] Benilan, P., Brezis, H. Solutions faibles d'équations d'évolution dans les espaces de Hilbert. Annales de l'Institut Fourier. 22 (1972), no. 2, 311-329.

[10] Bотне, D. Flow invariance for nonlinear accretive evolutions under range conditions. J. Evol. Equ. 5 (2005), no. 2, 227-252.

[11] Brezis, H. On a characterization of flow-invariant sets. Comm. Pure Appl. Math. 23 (1970) 261-263.

[12] Brezis, H. Opérateurs Maximaux Monotones et Semi-groupes de Contractions dans les Espaces de Hilbert. North-Holland, Amsterdam, 1973.

[13] Brezis, H. Analyse Fonctionnelle, ThÈorie et Applications., Masson, 1987.

[14] CÂrJaĂ, O., Motreanu, D. Flow-invariance and Lyapunov pairs. Dyn. Contin. Discrete Impuls. Syst. Ser. A Math. Anal. 13B (2006), suppl., 185-198.

[15] CÂrjă̆, O., Motreanu, D. Characterization of Lyapunov pairs in the nonlinear case and applications. Nonlinear Anal. 70 (2009), no. 1, 352-363.

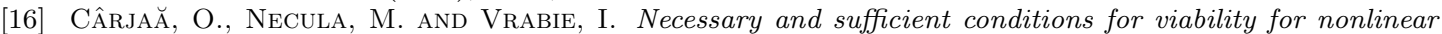
evolution inclusions. Set-Valued Anal. 16 (2008), 701-731.

[17] CÂrja $\breve{,}$, O., Necula, M. and Vrabie, I. Necessary and sufficient conditions for viability for semilinear differential inclusions. Trans. Amer. Math. Soc. 361 (2009), no. 1, 343-390.

[18] Chellaboina, V., Leonessa, A. and Haddad, W. M. Generalized Lyapunov and invariant set theorems for nonlinear dynamical systems. Systems Control Lett. 38 (1999), no. 4-5, 289-295.

[19] Clarke, F. H. Optimization and nonsmooth analysis. Second edition. Classics in Applied Mathematics, 5. Society for Industrial and Applied Mathematics (SIAM), Philadelphia, PA, 1990.

[20] Clarke, F. H., Ledyaev, Y. S., Stern, R. J. and Wolenski, P. R. Qualitative properties of trajectories of control systems: a survey. J. Dynam. Control Systems. 1 (1995), no. 1, 1-48.

[21] Clarke, F. H., Ledyaev, Y. S., Stern, R. J. and Wolenski, P. R. Nonsmooth analysis and control theory. Graduate Texts in Mathematics, 178. Springer-Verlag, New York, 1998.

[22] Ekeland I. On the variational principle, J. Math. Anal. and Appl., 47 (1974), 324-353.

[23] Goeleven, D., Motreanu, D. And Motreanu, V. V. On the stability of stationary solutions of first order evolution variational inequalities. Adv. Nonlinear Var. Inequal. 6 (2003), no. 1, 1-30.

[24] Kocan, M., Soravia, P. Nonlinear, dissipative, infinite dimensional systems, in "Stochastic Analysis, Control, Optimization, and Applications" (W. H. Fleming et al., Eds.) (2002), no. 2, 342-363.

[25] Kocan, M., Soravia, P. Lyapunov functions for infinite-dimensional systems. J. Funct. Anal. 192 (2002), no. 2, 342-363.

[26] Martinez-Legaz, J.-E., Théra, M. $\varepsilon$-subdifferentials in terms of subdifferentials. Set-Valued Anal. 4 (1996), no. 4, 327-332

[27] Mordukhovich, B. S. Variational analysis and generalized differentiation. I. Basic theory. Grundlehren der Mathematischen Wissenschaften [Fundamental Principles of Mathematical Sciences], 330. SpringerVerlag, Berlin, 2006.

[28] Mordukhovich, B. S., Shao Y. Nonsmooth sequential analysis in Asplund spaces,. Trans. Amer. Math. Soc. 348 (1996), 1235-1280.

[29] Pavel, N.H. Invariant sets for a class of semilinear equations of evolution. Nonlin. Anal. TMA. 1 (1977) $187-196$

[30] PAZY, A. The Lyapunov method for semigroups of nonlinear contractions in Banach spaces. J. Anal. Math. 40 (1981), 239-262.

[31] Rockafellar, R.T., Wets, R. J.-B. Variational analysis. Grundlehren der Mathematischen Wissenschaften [Fundamental Principles of Mathematical Sciences], 317. Springer-Verlag, Berlin, 1998.

[32] Shevitz, D., PAden, B. Lyapunov stability theory of nonsmooth systems. IEEE Trans. Automat. Control 39 (1994), no. 9, 1910-1914.

[33] Simons, S. Minimax and Monotonicity. Lecture Notes in Mathematics. Volume 1693, Springer-Verlag, Berlin, 1998.

[34] Smirnov, G. Introduction to the theory of differential inclusions. Graduate Studies in Mathematics, 41, American Mathematical Society, 2002.

[35] Tartar, L. Topics in Nonlinear Analysis. Publ. Math. Univ. d'Orsay,. 13 (1978).

[36] Vrabie, I. I. Nagumo viability theorem. Revisited. Nonlinear Anal. 64 (2006), no. 9, 2043-2052.

[37] Zhu, Q.J. Lower semicontinuous Lyapunov functions and stability. J. Nonlinear Convex Anal. 4 (2003), no. $3,325-332$. 